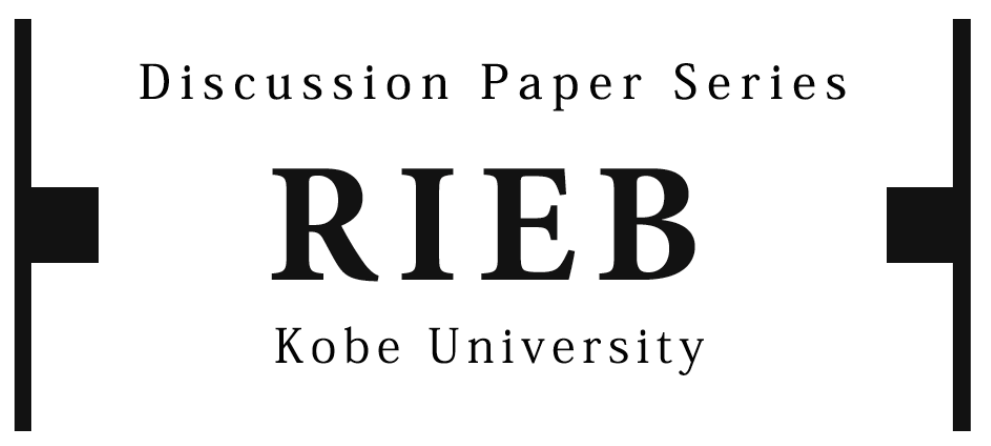

DP2021-15

\title{
The Bargaining Set and Coalition Formation
}

\author{
Ken-Ichi SHIMOMURA
}

June 21, 2021

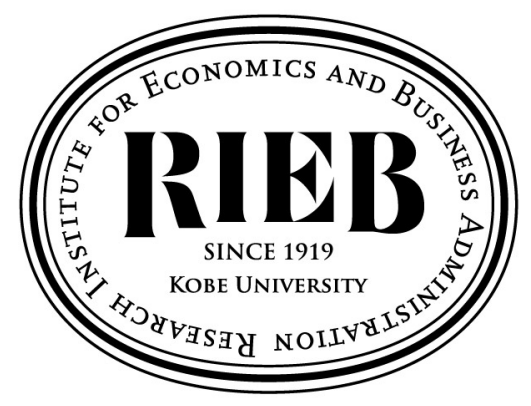

Research Institute for Economics and Business Administration Kobe University 


\title{
The Bargaining Set and Coalition Formation
}

\author{
Ken-Ichi Shimomura* \\ June, 2021 \\ Forthcoming in the International Journal of Economic Theory
}

\begin{abstract}
We address the problem of predicting how rational agents will form coalitions in a nontransferable utility game, and within each coalition how they will allocate the gains obtained through cooperation. To answer these questions, we propose solution concepts according to which the coalition structure and the payoff allocations are simultaneously determined. We prove the nonemptiness and partial efficiency of the steady bargaining set, a refinement of the Zhou bargaining set, for at least one coalition structure under the restrictive non-crossing condition. In addition, we show the nonemptiness and possible inefficiency of the Mas-Colell bargaining set if this condition is not assumed.
\end{abstract}

Keywords: Nontransferable utility game, coalition structure, bargaining set, restrictive noncrossing condition

JEL classification No: C71, D71

\section{Introduction}

We address the problem of predicting how rational agents, namely players, will form coalitions, and within coalitions how they will allocate the gains obtained through cooperation. We define a coalition structure as a partition of the whole set of players, and formulate the underlying situation as a coalitional game with coalition structure, in which the allocation of payoffs will depend on which coalitions actually form. We consider a coalition structure as a collection of coalitions whose members are always free to form a new coalition for higher payoffs. Then, the eventual formation

\footnotetext{
* RIEB, Kobe University, 2-1 Rokkodai, Nada, Kobe, Hyogo 657-8501, Japan.

(email: ken-ichi@rieb.kobe-u.ac.jp)

This is a revision of Brown University Economics Working Paper 95-11. I am grateful to Youngsub Chun for giving me the opportunity of a publication, and Ryo-ichi Nagahisa for calling my attention to the topic of the paper. Many helpful comments and discussions with Rajiv Vohra and William Thomson are greatly appreciated. Thanks are also due to the late Michael Maschler, Tatsuro Ichiishi, and Roberto Serrano for encouragement, suggestions and conversations. I am indebted to Barry Feldman for pointing out an error, and Matthew Coffey for reading carefully an earlier draft. This research is partially supported by Kyoto University Foundation, Kikawada Foundation, and the Japanese Ministry of Education, Culture, Sports, Science and Technology (Grants-in-aid for Scientific Research (A) 18H03641 and (C) 19K01558).
} 
of coalitions will be influenced by what players expect to get in different coalitions. We thus search for a solution in the family of bargaining sets to select the coalition structure and the payoff allocation simultaneously, and study the existence and the efficiency of solution outcomes.

The idea of the bargaining set starts from a study by Aumann and Maschler (1964) of a stable solution for the class of coalitional games with coalition structures. Their bargaining set $M_{1}^{(i)}$ is defined in terms of an "objection from a player to another player given a proposal" and a "counterobjection from the objected player to the objecting player." The notion of the bargaining set depends on the particular definitions of objections and counterobjections being considered. Several alternative definitions have been since. In this paper, we define an "objection from a coalition to a proposal" and a "counterobjection from an objected coalition to the objecting coalition." Here is the essence: Let $x$ be a recommended payoff profile. Then, a coalition $S$ has an objection if it can produce an alternative profile of payoffs for its members that make them strictly better off than at $x$. A coalition $T$ has a counterobjection to $S$ at $x$ if $T$ can achieve a payoff profile that makes its members at least as well off as in $x$, and makes players belonging to both $S$ and $T$ at least as well off as they are in the objection. An objection to $x$ is "justified" if it has no counterobjection.

Davis and Maschler (1963), and Peleg (1963) prove that, for transferable utility (TU) games, Aumann and Maschler's bargaining set $M_{1}^{(i)}$ is nonempty-valued for all coalition structures. Thus, $M_{1}^{(i)}$ is not a solution to select stable coalition structures. Aumann and Drèze (1974) investigate not only $M_{1}^{(i)}$ but also the core of a coalitional game with a coalition structure, which is the set of payoff profiles feasible for the coalition structure with no objections from any coalition. Aumann and Drèze show that the core of a balanced TU game with a coalition structure is nonempty if and only if the coalition structure maximizes the sum of the "worths" of the partitions of the grand coalition [For details, see Shimomura (1997, P.295)]. This tells that the core selects stable pairs of coalition structure and payoff allocation for balanced TU games, but it does not work for the other types of TU games.

For nontransferable utility (NTU) game, Mas-Colell (1989) proposes a modification of $M_{1}^{(i)}$, now called the Mas-Colell bargaining set. Mas-Colell's original is defined to be the set of efficient payoff profiles produced by the grand coalition with no justified objection. The Mas-Colell bargaining set of a coalitional game with a coalition structure, discussed in this paper, is the set of payoff profiles feasible for the coalition structure with no justified objection. In a TU game, it contains efficient payoff profiles for at least one coalition structure [Zhou (1994), Shimomura (1997)]. In fact, Mas-Colell (1989, P.138) points out that for many games, his bargaining set "can be quite large" regardless of the requirement of efficiency. Thus, the Mas-Colell bargaining set per $s e$ is not a solution to select stable payoff allocations.

To our best knowledge, there has been no research on the Mas-Colell bargaining set of NTU games with coalition structures although Vohra (1991) gives sufficient conditions for the nonemptiness, which implies efficiency, of Mas-Colell's original bargaining set of NTU games. In 
this paper, we investigate refinements of the Mas-Colell bargaining set to identify stable coalition structures and internal payoff allocations for a reasonably large class of NTU games including all TU games. Zhou (1994) is the first author to challenge this project. We pursue a better notion of stability for a bargaining set based on the fact that the outcomes become more sophisticated as the requirements imposed on counterobjection to qualify are strengthened.

To do so, we propose criterion to adopt counterobjections in the following ways. We first impose the intersection criterion, intensively investigated by Zhou (1994). In the definition of a counterobjection by Mas-Colell (1989) as well as Aumann and Maschler's (1964), a counterobjecting coalition may not include any member of the objecting coalition. That is, the coalitions making the objection and the counterobjection may have an empty intersection. Zhou (1994) points out the importance of this requirement on counterobjections of a nonempty intersection, what we call the "proper intersection condition." The resulting solution concept is the Zhou bargaining set. We will investigate how strong of an intersection requirement one can impose on counterobjections so as not to make the bargaining set empty for all coalition structures.

The second is the improvement criterion. Mas-Colell (1989) and Vohra (1991) define an objection and a counterobjection as follows: an alternative payoff vector to the recommendation $x$ in a coalition is proposed which keeps its members as well off as at $x$, and at least one of them strictly better off compared (and the objection to be counterobjected). Notice the symmetry in their definitions of objections and counterobjections: both are defined in terms of Pareto-improvement. We will examine the possibility of requring strict improvements, that is making all the members of the objecting and counterobjecting coalitions strictly better off than at $x$. Note that the concepts of objection-counterobjection defined by Pareto-improvement and strict improvement are not logically related.

The third criterion is the dominance criterion. Suppose that a payoff vector is proposed for a society, and coalition $S$ has an objection. Counterobjections are defined objection by objection; which coalition is against $S$ depends on the objection proposed by $S$. A coalition $T$ will be disinclined to counterobject if they know that $S$ has an alternate option to which $T$ has no counterobjection. The story is different if $T$ can make a counterobjection to every possible objection that $S$ can make against $x$. That is, $T$ can break any deviation by $S$ from $x$. We will require counterobjections to satisfy this criterion and define the steady bargaining set.

The steady bargaining set is contained in the Zhou bargaining set, which passes the intersection and dominance tests. We propose the subset of the steady bargaining set obtained by imposing the improvement criterion for both objections and counterobjections. We call this the strong steady bargaining set.

We discuss one more bargaining set. We refine the Mas-Colell bargaining set by requiring the objection to be in the core of the "reduced game" on the objecting coalition as well as both objections and counterobjections to be defined by strict improvements. We call it the precise bargaining set. The steady bargaining set is almost, but not quite, a subset of the precise bargaining 
set. The strong steady bargaining set is contained in both the precise bargaining set and the steady bargaining set.

We establish three theorems. First, for a NTU coalitional game satisfying the "restrictive noncrossing condition," the steady bargaining set contains at least one efficient payoff profile for at least one coalition structure. Second, for a NTU coalitional game satisfying a "comprehensiveness" assumption as well as the "restrictive non-crossing condition," the strong steady bargaining set contains at least one efficient payoff profile. The restrictive non-crossing condition, which all TU games satisfy, enables us to avoid the "balanced family" argument, which is used by Scarf (1967), Vohra (1991), and Zhou (1994). We also show by way of an example that the Zhou bargaining set may be empty and the Mas-Colell bargaining set may be inefficient without the restrictive noncrossing condition. As corollaries, we deduce the possible emptiness of the steady bargaining set. This tells us that a stable compromise does not generally lead to efficiency; it is a problem shared by coalitional games and strategic games. Third, on the class of all NTU coalitional games, the precise bargaining set is nonempty for at least one coalition structure although it may not contain any efficient payoff vectors. To prove this, we need essentially no assumptions. The only condition used in our proof is that for every coalition, the set of all individually rational payoff profiles is compact.

Zhou (1994) shows that on the class of TU games, the Zhou bargaining set contains at least one efficient payoff profile for at least one coalition structure. Our first theorem extends his result to NTU games similarly to the way Scarf (1967) had extended the core nonemptiness theorem of Bondareva (1963) and Shapley (1967). Shimomura (1997) proves that on the class of TU games, the steady bargaining set and the Zhou bargaining set are nonempty for every coalition structure that can produce the maximal sum of payoffs to all the players. Combining this result with our first theorem, we demonstrate that in a NTU coalitional game, it is not easily predictable which coalitions are likely to form, but the idea of the bargaining set helps explain how coalition structures and payoff allocations are determined.

The paper is organized as follows. In Section 2, we present a model of an NTU coalitional game and define the Mas-Colell bargaining set. In Section 3, we propose, as refinements of the MasColell bargaining set, the Zhou bargaining set, the steady bargaining set, and the strong steady bargaining set, and investigate their nonemptiness and efficiency. In Section 4, we propose two subsolutions of the Mas-Colell bargaining set, which are the precise bargaining set and the natural bargaining set, and investigate their nonemptiness. Section 5 is for concluding remarks. The appendix provides the proofs of the results stated in Sections 3 and 4.

\section{The Model and the Mas-Colell Bargaining Set}

In this section, we define fundamental concepts underlying the bargaining set in the model of a coalitional game. The set of players is a nonempty finite set denoted by $N$, and a coalition is a nonempty subset of $N$. For every coalition $S$, a payoff profile for $S$ is a point of $\mathbb{R}^{S}$ (throughout the paper, we denote by $X^{A}$ the set of functions from the set $A$ to the set $X$. If $A$ is finite, then every point $g \in X^{A}$ is identified with the bundle $\left.(g(a))_{a \in A}\right)$. We simply call a payoff profile for $N$ a payoff 
profile. Given a payoff profile $x$, we denote by $x_{i} \equiv x(i)$ the payoff to player $i$ at profile $x$, and by $x_{S}$ the projection of $x$ on $\mathbb{R}^{S}$. For each $B \subset \mathbb{R}^{S}$, we denote by int $B$ and $\operatorname{bd} B$ respectively the interior and the boundary of $B$ relative to $\mathbb{R}^{S}$. Let $S \subset N$, and $F \subset \mathbb{R}^{S}$. A representation for $F$ is a function $f: \mathbb{R}^{S} \rightarrow \mathbb{R}$ such that $F=\left\{x \in \mathbb{R}^{S} \mid f(x) \leq 0\right\}$ and int $F=\left\{x \in \mathbb{R}^{S} \mid f(x)<0\right\}$. We define the following concepts:

Coalitional Game: A coalitional game, simply a game, is a correspondence $V$ that associates with every coalition $S$ a set $V(S) \equiv\left\{x \in \mathbb{R}^{N} \mid x_{S} \in V_{S}\right\}$, where $V_{S} \subset \mathbb{R}^{S}$, satisfying

continuity: there is a representation for $V_{S}$ that is continuous.

Moreover, there is a point $\underline{x} \in \mathbb{R}^{N}$ such that for every coalition $S$, the following hold:

x-compactness: $V_{S} \cap\left(\left\{\underline{x}_{S}\right\}+\mathbb{R}_{+}^{S}\right)$ is compact;

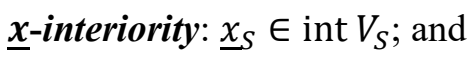

$\underline{\boldsymbol{x}}$-compehensiveness: for every $\left(x_{S}, y_{S}\right) \in \mathbb{R}^{S} \times V_{S}, \underline{x}_{S} \leq x_{S} \ll y_{S}{ }^{1} \Rightarrow x_{S} \in \operatorname{int} V_{S}$

Note that $V(S) \subset \mathbb{R}^{N}$ and $V_{S} \subset \mathbb{R}^{S}$. The point $\underline{x}$ plays a role similar to the reference point in cooperative bargaining games à la Nash. We interpret $\underline{x}$ as a profile of reservation payoffs. Then, $\underline{x}$ interiority corresponds to the "survival condition" in a general equilibrium model: every consumer can reduce his utility and still live by discarding part of his endowment.

We assume the continuity of representations to deal with games that are possibly bounded (such as games with $V_{S}$ compact) and simplify the proofs. Continuity implies that every $V_{S}$ is a closed subset of $\mathbb{R}^{S}$. If $V_{S}$ is comprehensive (i.e., $V_{S}-\mathbb{R}_{+}^{S} \subset V_{S}$ ) and non-leveled (i.e., for every $\left.\left(x_{S}, y_{S}\right) \in \operatorname{bd} V_{S} \times \mathrm{bd} V_{S}, x_{S} \leq y_{S} \Rightarrow x_{S}=y_{S}\right)$, then $V_{S}$ is $\underline{x}$-compehensive. Then for every coalition $S$, the frontier of $V_{S}$ is a connected surface. Note that, for every $y_{S} \in V_{S}$, the segment connecting $y_{S}$ with $\underline{x}_{S},\left[\underline{x}_{S}, y_{S}\left[\equiv\left\{x_{S} \in V_{S} \mid\left(\exists t \in\left[0,1[)\left(x_{S}=(1-t) \underline{x}_{S}+y_{S}\right)\right\}\right.\right.\right.\right.$, is included in $\operatorname{int} V_{S}$, namely $\left[\underline{x}_{S}, y_{S}\left[\subset \operatorname{int} V_{S}\right.\right.$. By continuity, $\underline{x}$-comprehensiveness implies:

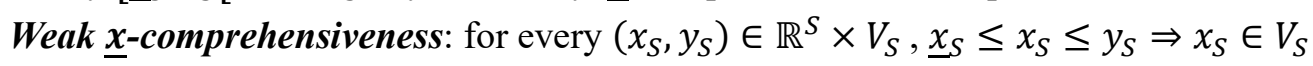

The converse is not always true: weak $\underline{x}$-comprehensiveness does not imply $\underline{x}$-comprehensiveness. In addition, $\underline{x}$-comprehensiveness is weaker than the following:

Strict $\underline{x}$-comprehensiveness: for every coalition $S$ and $\left(x_{S}, y_{S}\right) \in \mathbb{R}^{S} \times V_{S}$, $\underline{x}_{S} \leq x_{S} \ll y_{S} \& x_{S} \neq y_{S} \Rightarrow x_{S} \in \operatorname{int} V_{S}$

A coalition structure is a partition of $N$. We denote by $\Pi$ the class of all coalition structures. Let $V$ be a game. The feasible set for coalition structure $\mathcal{P}$ is $V^{*}(\mathcal{P}) \equiv\left\{x \in \mathbb{R}^{N} \mid \forall P \in \mathcal{P}, x_{P} \in V_{P}\right\}=$ $\bigcap_{P \in \mathcal{P}} V(P)$, and the feasible set of $V$ is $V^{*} \equiv \cup_{\mathcal{P} \in \Pi} V^{*}(\mathcal{P})$. For every coalition $S$, a payoff profile $x_{S}$ for $S$ is individually rational if $x_{i} \geq \sup V_{\{i\}}$ for all $i \in S$. The individually rational set for the coalition structure $\mathcal{P}$ is $I(V ; \mathcal{P}) \equiv\left\{x \in V^{*}(\mathcal{P}) \mid \forall i \in N, x_{i} \geq \sup V_{\{i\}}\right\}$, and the individually rational set of $V$ is $I(V) \equiv \cup_{\mathcal{P} \in \Pi} I(V ; \mathcal{P})$. A payoff profile $x$ is efficient if $x \in V^{*}$, and there is no $y \in V^{*}$ such that $y_{i}>x_{i}$ for all $i \in N$. A coalition structure $\mathcal{P}$ is efficient if there is at least one individually rational and efficient payoff profile $x \in V^{*}(\mathcal{P})$.

\footnotetext{
${ }^{1}$ Vector inequalities: $x \leq y$ if $x_{i} \leq y_{i}$ for all $i, x \ll y$ if $x_{i}<y_{i}$ for all $i$.
} 
A transferable utility (TU) game is a game $V$ such that for every coalition $S$, there is a real number $v(S)$ such that $V_{S}=\left\{x \in \mathbb{R}^{S} \mid \sum_{i \in S} x_{i} \leq v(S)\right\}$. The number $v(S)$ is the worth of $S$.

Let $S$ and $T$ be two coalitions, $x$ a payoff profile, $y \in V_{S}$, and $z \in V_{T}$. Then the pair $(T, z)$ weakly improves upon $(S, y)$ at $x$ if $(T, z) \neq(S, y)$ and $z \geq\left(x_{T \backslash S}, y_{T \cap S}\right)$; it Pareto-improves upon $(S, y)$ at $x$ if $z \geq\left(x_{T \backslash S}, y_{T \cap S}\right)$ and $z \neq\left(x_{T \backslash S}, y_{T \cap S}\right)$; and it strictly improves upon $(S, y)$ at $x$ if $z \gg\left(x_{T \backslash S}, y_{T \cap S}\right)$. If $(T, z)$ is such that $z \gg x_{T}$, then $(T, z)$ strictly improves upon $x$. Note that $(T, z)$ weakly improves upon $(S, y)$ at $x$ even if $(T, z) \neq(S, y)$ and $z=\left(x_{T \backslash S}, y_{T \cap S}\right)$.

With these terms, we define the following concepts.

Objection: Let $V$ be a game and $x$ a payoff profile. Then the pair $(S, y)$ is an objection to $x$ if $S$ is a coalition and $y \in V_{S}$ strictly improves upon $x$.

The Core: The core of a game $V$ for coalition structure $\mathcal{P}$ is:

$C_{0}(V ; \mathcal{P})=\{x \in I(V ; \mathcal{P}) \mid$ There is no objection to $x\}$.

The core of $V$ is: $C_{0}(V)=\cup_{\mathcal{P} \in \Pi} C_{0}(V ; \mathcal{P})$.

This is the definition of the core of a general coalitional game in terms of strict improvement [Scarf (1967), and Peleg (1985)]. Scarf(1967) proves a nonemptiness theorem for the core of a "balanced" game with coalition structure $\{N\}{ }^{2}$

Counterobjection: Let $V$ be a game, $x$ a payoff profile, and $(S, y)$ an objection to $x$. Then the pair $(T, z)$ is a counterobjection to $(S, y)$ at $x$ if $T$ is a coalition and $z \in V_{T}$ weakly improves upon $(S, y)$ at $x$.

Mas-Colell bargaining set: The Mas-Colell bargaining set of a game $V$ for coalition structure $\mathcal{P}$ is: $M B(V ; \mathcal{P})=\{x \in I(V ; \mathcal{P}) \mid$ For each objection to $x$, there is a counterobjection at $x\}$.

The Mas-Colell bargaining set of $V$ is: $M B(V)=\cup_{\mathcal{P} \in \Pi} M B(V ; \mathcal{P})$.

Our definition of MB differs from Mas-Colell's (1989, Section 6) original definition. Mas-Colell does not require individually rationality, and defines both objections and counterobjections by Pareto-improvement within coalitions ${ }^{3}$. We impose individual rationality constraint on $M B$ and define counterobjections by weak improvement of counterobjecting coalitions. This is similar to the definition of the classical bargaining set $M_{1}^{(i)}$, proposed by Aumann and Maschler (1964) [see also Aumann (1989, P.77)]: Aumann and Maschler impose individual rationality constraint on $M_{1}^{(i)}$.

\footnotetext{
${ }^{2}$ A class $\mathcal{F}$ of coalitions is a balanced family if there is a function $\delta$ that assigns to each coalition $S$ a real number $\delta(S)$ such that $\delta(S)>0$ for $S \in \mathcal{F}, \delta(S)=0$ for $S \notin \mathcal{F}$, and $\sum_{S \in \mathcal{F}(i)} \delta(S)=1$, where $\mathcal{F}(i)=\{S \in \mathcal{F} \mid S \ni i\}$ for every player $i$. A game $V$ with coalition structure $\mathcal{P}$ is balanced if $\bigcap_{S \in \mathcal{F}} V(S) \subset \bigcap_{P \in \mathcal{P}} V(P)$ for every balanced family $\mathcal{F}$.

${ }^{3}$ Mas-Colell (1989, Section 1) defines counterobjections by strict improvement in atomless economies. He says that it "cannot simply be replaced by" weak improvement for the equivalence between his bargaining set and the set of Walrasian allocations in this environment.
} 
They define an "objection of player $i$ against player $j$ at $x$ " as strict improvement on $i$ via coalition $S$ such that $i \in S$ and $j \notin S$ without making any members of $S$ worse off than at $x$. They also define a "counterobjection from player $j$ to player $i$ " as weak improvement on $j$ via coalition $T$ such that $j \in T$ and $i \notin T$ without hurting any members of $T \backslash S$ or $T \cap S$ than at $x$ or at the objection via $S$.

\section{The Zhou Bargaining Set and the Steady Bargaining Set}

In this section, we develop the refinement of the Mas-Colell bargaining set proposed by Zhou (1994) in the NTU case. According to the above definition, a counterobjecting coalition may include no player in the objecting coalition. This means that the counterobjection is not really against the objection but the original proposal. To overcome this weakness, Zhou then proposes to require the intersection of the objection coalition and the counterobecting coalition to be a nonempty proper subset of the two coalitions respectively to overcome this weakness. Based on this, Zhou defines a refined bargaining set now called the "Zhou bargaining set."

Further, we introduce a dominance relation between coalitions to identify powerful counterobjections. Given an initial recommendation, which coalitions have counterobjections to an objection depends on what payoffs can be alternatively assigned to the members of the objecting coalition. We select as "dominant" those coalitions having a counterobjection to every objection from the objecting coalition. We impose on counterobjections both Zhou's intersection condition and the dominance requirement. We then introduce the corresponding version, the "steady bargaining set," as a refinement of the Zhou bargaining set. We establish its nonemptiness under the "restrictive non-crossing condition. "In addition, we remove the asymmetry between the improvement requirement underlying the objections and counterobjections. We select counterobjections which strictly improve upon the counterobjecting coalitions, and focus on the associated subset of the steady bargaining set. This is the "strong steady bargaining set." We prove its nonemptiness under the assumption of "strict $\underline{x}$-comprehensiveness."

Since the introduction of the bargaining set by Aumann and Maschler (1964), no intersection requirement has appeared in the definitions of alternative versions of the bargaining set, including $M B$ [see Zhou (1994, Sections 2 and 3) and Iñarra et al.. (2020, pp.254-253) for details]. Zhou (1994) proposes the following modification of the Mas-Colell bargaining set.

Proper Intersection/Proper counterobjection: Let $V$ be a game, and $S, T \subset N$. Then, $T$ has a proper intersection with $S$ if $T \cap S \neq \varnothing, S \backslash T \neq \varnothing$, and $T \backslash S \neq \varnothing$. Let $x$ be a payoff profile, $(S, y)$ an objection to $x$, and $(T, z)$ a counterobjection to $(S, y)$ at $x$. Then, $(T, z)$ is proper if $T$ has a proper intersection with $S$.

Zhou bargaining set: The Zhou bargaining set of a game $V$ for coalition structure $\mathcal{P}$ is: $Z B(V ; \mathcal{P})=\{x \in I(V ; \mathcal{P}) \mid$ For each objection $(S, y)$ to $x$, there is a proper counterobjection to $(S, y)$ at $x\}$ The Zhou bargaining set of $V$ is: $Z B(V)=\bigcup_{\mathcal{P} \in \Pi} Z B(V ; \mathcal{P})$.

Figure 1: Coalition $T$ having a proper intersection with coalition $S$

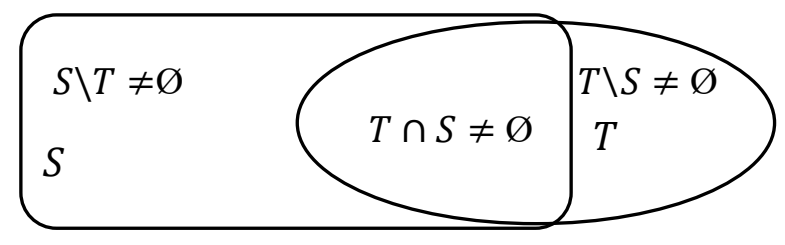


Figure 1 illustrates coalitions $S$ and $T$ having a proper intersection. This captures the idea that some players outside of $S$ tempt some members of $S$ to form a new coalition $T$. For the objection made by $S$ to be valid, the players in $T \cap S$ should not have an incentive to move to $T$.

We now propose a refinement of the Zhou bargaining set by invoking a dominance relation between coalitions: Consider a situation that, given a game $V$ and a coalition structure $\mathcal{P}$, a payoff profile $x$ is proposed. Suppose that some players are not satisfied. They form a coalition $S$, not in $\mathcal{P}$, and formulate an objection to $x$. According to the Zhou bargaining set, the objection is justified unless at least one counterobjection is made by a coalition having a proper intersection with $S$. Then another coalition $T$ cannot neutralize $S$ if $S$ propose payoffs for its members to which $T$ can find no counterobjection at $x$. Our idea is that $S$ will never form if $T$ can make a counterobjection to every possible objection to $x$ that $S$ could make. We then say that " $T$ dominates $S$ at $x$." Based on this relation between coalitions, we define a new solution to assign to each game a payoff profile to which an objecting coalition can form if and only if it is not "dominated" by any other coalition.

Dominating coalition: Let $V$ be a game, $x$ a payoff profile, and $S, T$ coalitions. Then, $T$ dominates $S$ at $x$ if for each objection $(S, y)$ to $x$, there is a proper counterobjection $(T, z)$ to $(S, y)$ at $x$.

Steady bargaining set: The steady bargaining set of a game $V$ for coalition structure $\mathcal{P}$ is: $S B(V, \mathcal{P})=\{x \in I(V ; \mathcal{P}) \mid$ For each objection to $x$, there is a dominant coalition at $x\}$. The steady bargaining set of a game $V$ is: $S B(V)=\cup_{\mathcal{P} \in \Pi} S B(V ; \mathcal{P})$

For every game $V$, the inclusion relation among the core, the Mas-Colell bargaining set, the Zhou bargaining set, and the steady bargaining set is following: $C_{0}(V ; \mathcal{P}) \subset S B(V ; \mathcal{P}) \subset Z B(V ; \mathcal{P}) \subset$ $M B(V ; \mathcal{P})$ for every coalition structure $\mathcal{P}$, and $C_{0}(V) \subset S S B(V) \subset S B(V) \subset Z B(V) \subset M B(V)$. The steady bargaining set is generally a proper subset of the Zhou bargaining set. For example, let $V$ be the three-person TU game such that the worth of $S$ is 0 if $|S|=1, a$ if $|S|=2$, and 1 if $|S|=3$. Then $S B(V ;\{N\})$ is a proper subset of $Z B(V ;\{N\})$ if $2 / 3<a \leq 1$ [see Shimomura (1997, Section 4) for details]. The Zhou bargaining set is also generally a proper subset of the Mas-Colell bargaining set. Zhou (1994, Example 3.2) presents a TU game of which the Zhou bargaining set does not contain the nucleolus while the Mas-Colell bargaining set does [see also Shimomura (1997, Example 4)]. We exhibit a game in which the Zhou bargaining set is empty and the Mas-Colell bargaining set is nonempty later (Example 2). To compare properties of these two solutions, we impose a new assumption on a game together with the basic four in the definition.

Restrictive non-crossing condition: For every $x \in P\left(V^{*}\right)$, there is no triple $(S, R, Q)$ of disjoint coalitions, and two profiles $y_{S}, y_{S}^{\prime} \in\left[\left\{x_{S}\right\}+\mathbb{R}_{++}^{S}\right] \cap\left[\left\{r_{S}\right\}+\mathbb{R}_{++}^{S}\right]$ such that

$$
\left(y_{S}, x_{R}\right) \in \operatorname{int} V_{S \cup R} \&\left(y_{S}, x_{Q}\right) \notin V_{S \cup Q} \&\left(y_{S}{ }^{\prime}, x_{Q}\right) \in \operatorname{int} V_{S \cup Q} \&\left(y_{S}{ }^{\prime}, x_{R}\right) \notin V_{S \cup R}
$$

where $P\left(V^{*}\right) \equiv\left\{x \in V^{*} \cap\left[\{\underline{x}\}+\mathbb{R}_{+}^{N}\right] \mid\right.$ There is no $y \in V^{*}$ such that $\left.y \gg x\right\}$ and $r_{S} \equiv\left(\sup V_{\{i\}}\right)_{i \in S}$. 
This condition says the following: given an efficient payoff vector $x$, suppose that the coalition $S$ can achieve new payoffs in collaboration with $R$ but not $Q$ at which all the members of $S$ are strictly better off than at $x$ and at the individually rational levels. Then $S$ can realize with $R$ any such payoffs that can be produced with $Q$. See Figure $2 .{ }^{4}$ The top panel represents a game that satisfies the restrictive non-crossing condition, and the bottom panel describes a game that does not satisfy. Vohra (1991) points out the importance of almost, but not exactly, the same condition for a game to have an efficient payoff profile in the Mas-Colell bargaining set. He also gives pictures as in Figure 2 to illustrate a case in which the condition is satisfied and a case in which it is not satisfied; the frontiers of the slices, $V_{S}^{x_{R}}(S \cup R) \equiv\left\{y_{S} \in \mathbb{R}^{S} \mid\left(y_{S}, x_{R}\right) \in V_{S \cup R}\right\}$ and $V_{S}^{x_{Q}}(S \cup Q) \equiv$ $\left\{y_{S} \in \mathbb{R}^{S} \mid\left(y_{S}, x_{Q}\right) \in V_{S \cup Q}\right\}$ cross.

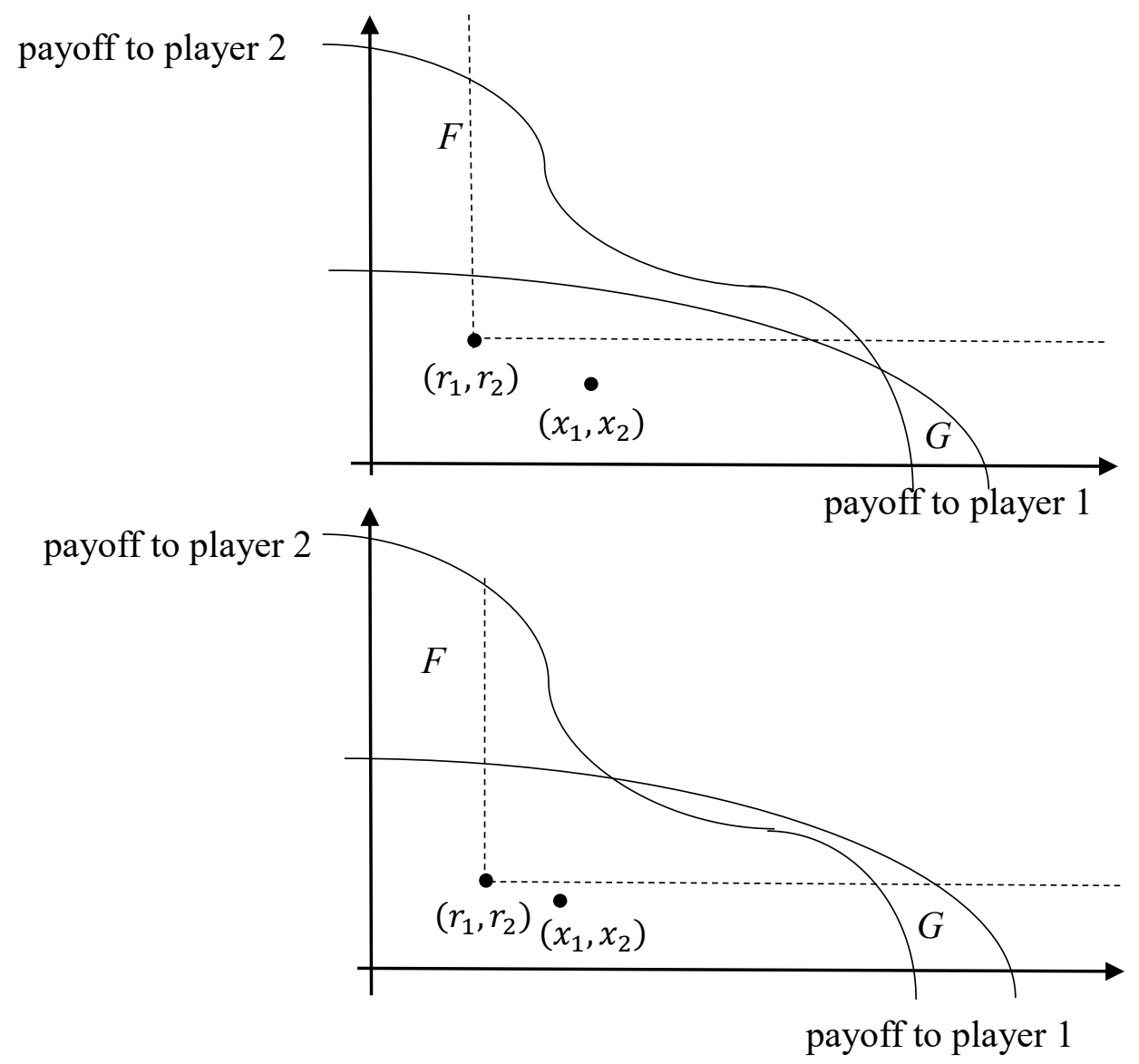

Figure 2: Game that satisfies the restrictive non-crossing condition and one that does not, where $S=\{1,2\}, x \in P\left(V^{*}\right), F=V_{S}^{x_{R}}(S \cup R)$, and $G=V_{S}^{x_{Q}}(S \cup Q)$

\footnotetext{
${ }^{4}$ Demuynck and Potoms (2020) propose essentially the same condition for utility possibility sets named the "no intersection property."
} 
Notice the restriction " $x \in P\left(V^{*}\right)$." The restrictive non-crossing condition does not say anything about the frontiers of slices through inefficient payoff profiles. If the feasible set $V^{*}$ is large enough and payoffs $x \in P\left(V^{*}\right)$ are too large, we may have either $\left(x_{S}, x_{R}\right) \notin \operatorname{int} V_{S \cup R}$ or $\left(r_{S}, x_{R}\right) \notin$ $\operatorname{int} V_{S \cup R}$ as long as $S \cup R \neq N$. Then the restrictive non-crossing condition is trivially satisfied even though the frontiers of the slices through some inefficient profiles may be crossing.

All TU games and all three-person comprehensive games satisfy the restrictive non-crossing condition. A comprehensive game $V$ such that $V(S) \subset \bigcap_{i \in S} V(\{i\})$ for every $S \subset N$ with $|S| \geq 3$ and $S \neq N$ also satisfies the condition. These are games in which the two-person coalitions are essential. "Roommate problems" are representable as such games, and may have an empty core [see Shubik (1984, p.221)]. We now examine the nonemptiness of the bargaining sets of a TU coalitional game with coalition structures. Zhou (1994) proves the following theorem.

Theorem (Zhou, 1994): The Zhou Bargaining Set of a TU game contains at least one efficient payoff profile for at least one coalition structure.

Zhou presents this theorem as an answer to the question of how players form coalitions. Our study is inspired by his work. We present the following result to which Zhou's theorem is a corollary:

Theorem 1: Given a game satisfying the non-crossing condition, the Steady Bargaining Set contains at least one efficient payoff profile for at least one coalition structure.

We prove Theorem 1 in the appendix. By Theorem 3.2 in Shimomura (1997), we know the following: Let $\mathcal{P}$ be a coalition structure such that $\max \left\{\sum_{i \in N} x_{i} \mid x \in V^{*}(\mathcal{P})\right\} \geq$ $\max \left\{\sum_{i \in N} x_{i} \mid x \in V^{*}(\mathcal{Q})\right\}$ for all coalition structures $\mathcal{Q}$. If $V$ is a TU game, then the steady bargaining set contains at least one efficient payoff profile for coalition structure $\mathcal{P}$. Call the value of $\max \left\{\sum_{i \in N} x_{i} \mid x \in V^{*}(\mathcal{Q})\right\}$ the worth of coalition structure $\mathcal{Q}$. If a coalition structure produces maximal worth in a game, it is efficient and it admits a nonempty steady bargaining set. However, this holds only in the TU case. The following example shows an NTU game admitting a worthmaximizing coalition structure such that even the associated Zhou bargaining set is empty.

Example 1. A Couple and a Friend on Sunday. There are two women and a man: Andy, Beth, and Olive. We denote them by $a, b$, and $o$, respectively. The agents $a$ and $b$ are lovers, and $o$ is their friend. The problem is how to spend the next Sunday. The (maximal) payoff each of them can achieve alone is zero. When $a$ and $b$ are together, they can achieve $(3,3)$. When $a$ and $o$, or $b$ and $o$, are together, they can achieve $(1,1)$. When they all are together, they can achieve $(2,2,2)$. Let $N=\{a, b, o\}$. Then the game $V$ describing the above situations is as follows:

$$
\begin{aligned}
& V_{\{i\}}=\left\{x_{i} \in \mathbb{R}^{\{i\}} \mid x_{i} \leq 0\right\} \text { for every } i \in N, \\
& V_{\{a, b\}}=\left\{x_{\{a, b\}} \in \mathbb{R}^{\{a, b\}} \mid\left(x_{a}, x_{b}\right) \leq(3,3)\right\},
\end{aligned}
$$




$$
\begin{aligned}
& V_{\{i, o\}}=\left\{x_{\{i, o\}} \in \mathbb{R}^{\{i, o\}} \mid\left(x_{i}, x_{o}\right) \leq(1,1)\right\} \text { for every } i \in\{a, b\}, \text { and } \\
& V_{N}=\left\{x \in \mathbb{R}^{N} \mid\left(x_{a}, x_{b}, x_{o}\right) \leq(2,2,2)\right\} .
\end{aligned}
$$

The efficient coalition structures are $[\{a, b\},\{o\}]$, and $[N]$, which are also worth-maximizing. The payoff profile $\left(x_{a}, x_{b}, x_{o}\right)=(3,3,0)$, which is achieved in the coalition structure $[\{a, b\},\{o\}]$, has no objection. Then it belongs to $Z B(V ;\{a, b\},\{o\})$ as well as to $C_{0}(V ;\{a, b\},\{o\})$. On the other hand, $(\{a, b\},(3,3))$ is an objection to any payoff vector of $V_{N}$ with no counterobjection. Then, $Z B(V ; N)$ as well as $M B(V ; N)$ is empty (if payoffs are transferable in $N$, i.e., $V_{N}=$ $\left\{x \in \mathbb{R}^{N} \mid x_{a}+x_{b}+x_{o} \leq 2+2+2\right\},(3,3,0)$ also belongs to $C_{0}(V ; N)$ and to $\left.Z B(V ; N)\right)$.

To establish a nonemptiness theorem for $M B(V ; N)$, Vohra (1991) proposes the assumption of "weak balancedness," which is implied by the restrictive no-crossing condition if the game $V$ satisfies strict $\underline{x}$-comprehensiveness. This assumption is weak, but as observed by Vohra, it may not be easy to verify by itself since the concept of a "justified objection" appears in the definition. Chang and Lee (1993) construct an example to show that weak balancedness is not necessary. Its counterpart here is stated as follows: "for every $x \in P\left(V^{*}\right) \cap\left[\{\underline{x}\}+\mathbb{R}_{+}^{N}\right]$, there is no pair $\left(S, S^{\prime}\right)$ of distinct maximal coalitions having proper counterobjections such that $S \cap S^{\prime} \neq \varnothing$." If we do not impose such a restriction on a game, not only can the Zhou bargaining set be empty, but also the Mas-Colell bargaining set may be inefficient. This means that some restrictions related to weak balancedness has to be included for the theorem. See the following example.

Example 2: Four Researchers and a Research Project. There are four researchers: Alex, Billie, Cameron, and Darian. We denote them by $a, b, c$ and $d$, respectively. They are involved in a research project. It is not possible for any one of them or any two of them to carry it out. If three of them cooperate, they have two plans from which to choose. Every agent has a preference over options of whom to work with and how to divide the job. Let $N=\{a, b, c, d\}$.

\begin{tabular}{|c|c|c|c|c|}
\hline coalition $\backslash$ agents & $a=$ Alex & $b=$ Billie & $c=$ Cameron & $d=$ Darian \\
\hline$\{a, b, c\}$ :plan 1 & 6 & 4 & 1 & - \\
\hline$\{a, b, c\}:$ plan 2 & 3 & 5 & 2 & - \\
\hline$\{b, c, d\}$ :plan 1 & - & 6 & 4 & 1 \\
\hline$\{b, c, d\}$ :plan 2 & - & 3 & 5 & 2 \\
\hline$\{a, c, d\}$ :plan 1 & 1 & - & 6 & 4 \\
\hline$\{a, c, d\}:$ plan 2 & 2 & - & 3 & 5 \\
\hline$\{a, b, d\}:$ plan 1 & 4 & 1 & - & 3 \\
\hline$\{a, b, d\}:$ plan 2 & 5 & 2 & - & 1.5 \\
\hline$N=\{a, b, c, d\}$ & 1.5 & 1.5 & 1.5 & 0.5 \\
\hline others & 0.5 & 0.5 & 0.5 & 6 \\
\hline
\end{tabular}

Table 1: Maximal payoffs players can get through cooperation 
Table 1 gives payoffs associated with the preferences. Take $\mathbf{0}=(0,0,0,0)$ as $\underline{x}$. The game $V$ defined below represents the feasible sets of payoff profiles:

$$
\begin{aligned}
& V_{\{a, b, c\}}=\left\{x_{\{a, b, c\}} \in \mathbb{R}^{\{a, b, c\}} \mid\left(x_{a}, x_{b}, x_{c}\right) \leq(6,4,1)\right\} \cup\left\{x_{\{a, b, c\}} \in \mathbb{R}^{\{a, b, c\}} \mid\left(x_{a}, x_{b}, x_{c}\right) \leq(3,5,2)\right\} \\
& V_{\{b, c, d\}}=\left\{x_{\{b, c, d\}} \in \mathbb{R}^{\{b, c, d\}} \mid\left(x_{b}, x_{c}, x_{d}\right) \leq(6,4,1)\right\} \cup\left\{x_{\{b, c, d\}} \in \mathbb{R}^{\{b, c, d\}} \mid\left(x_{b}, x_{c}, x_{d}\right) \leq(3,5,2)\right\} \\
& V_{\{a, c, d\}}=\left\{x_{\{a, c, d\}} \in \mathbb{R}^{\{a, c, d\}} \mid\left(x_{a}, x_{c}, x_{d}\right) \leq(1,6,4)\right\} \cup\left\{x_{\{a, c, d\}} \in \mathbb{R}^{\{a, c, d\}} \mid\left(x_{b}, x_{c}, x_{d}\right) \leq(2,3,5)\right\} \\
& V_{\{a, b, d\}}=\left\{x_{\{a, b, d\}} \in \mathbb{R}^{\{b, c, d\}} \mid\left(x_{b}, x_{c}, x_{d}\right) \leq(6,4,1)\right\} \cup\left\{x_{\{b, c, d\}} \in \mathbb{R}^{\{a, b, c\}} \mid\left(x_{b}, x_{c}, x_{d}\right) \leq(3,5,2)\right\} \\
& V_{N}=\left\{x \in \mathbb{R}^{N} \mid\left(x_{a}, x_{b}, x_{c}, x_{d}\right) \leq(1.5,1.5,1.5,1.5)\right\}, \text { and } \\
& V_{S}=\left\{x \in \mathbb{R}^{S} \mid x_{i} \leq 0.5 \text { for every } i \in S\right\} \text { for other coalitions } S .
\end{aligned}
$$

The game $V$ does not satisfy the restrictive non-crossing condition: let $x=(1.5,1.5,1.5,1.5) \in$ $V(N)$. We can verify $x \in P\left(V^{*}\right)$. Note that

$$
\begin{gathered}
V_{\{a, b\}}^{x_{c}}(\{a, b, c\})=\left\{y_{\{a, b\}} \in \mathbb{R}^{\{a, b\}} \mid\left(y_{a}, y_{b}\right) \leq(3,5)\right\}, \text { and } \\
V_{\{a, b\}}^{x_{d}}(\{a, b, d\})=\left\{y_{\{a, b\}} \in \mathbb{R}^{\{a, b\}} \mid\left(y_{a}, y_{b}\right) \leq(5,2)\right\}
\end{gathered}
$$

payoff to player

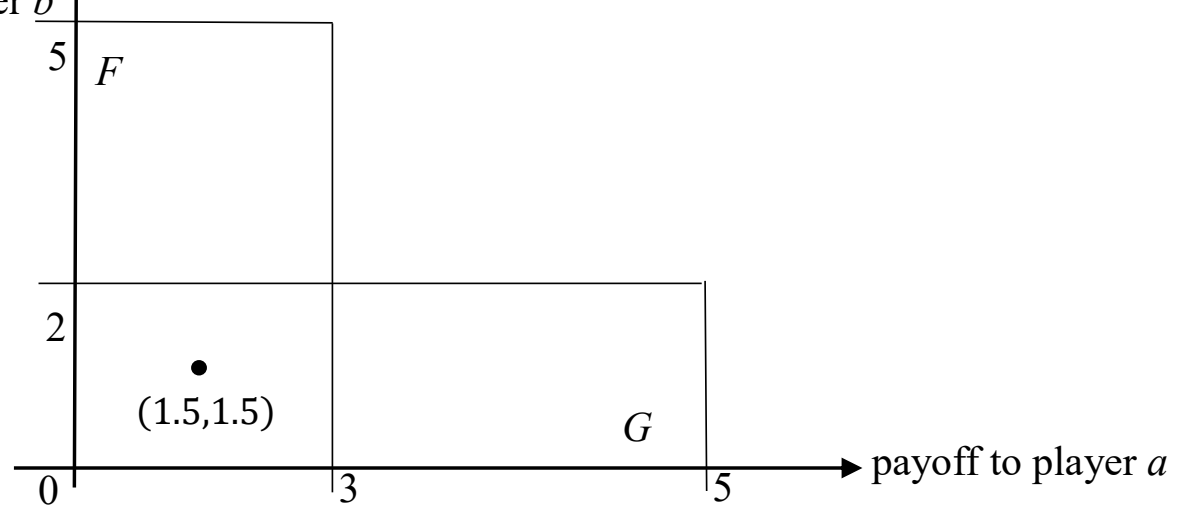

Figure 3: Slices of $V(\{a, b, c\})$ with $x_{c}$ fixed and $V(\{a, b, d\})$ with $x_{d}$ fixed

$$
S=\{a, b\}, x \in P\left(V^{*}\right), F=V_{\{a, b\}}^{x_{c}}(\{a, b, c\}), G=V_{\{a, b\}}^{x_{d}}(\{a, b, d\})
$$

These "slices" are drawn as in Figure 3. We see that

$$
\begin{aligned}
& V_{\{a, b\}}^{x_{c}}(\{a, b, c\}) \cap\left[\left\{x_{\{a, b\}}\right\}+\mathbb{R}_{++}^{\{a, b\}}\right] \cap\left[\left\{r_{\{a, b\}}\right\}+\mathbb{R}_{++}^{\{a, b\}}\right] \backslash V_{\{a, b\}}^{x_{d}}(\{a, b, d\}) \neq \varnothing, \text { and } \\
& V_{\{a, b\}}^{x_{d}}(\{a, b, d\}) \cap\left[\left\{x_{\{a, b\}}\right\}+\mathbb{R}_{++}^{\{a, b\}}\right] \cap\left[\left\{r_{\{a, b\}}\right\}+\mathbb{R}_{++}^{\{a, b\}}\right] \backslash V_{\{a, b\}}^{x_{c}}(\{a, b, c\}) \neq \varnothing
\end{aligned}
$$

The efficient coalition structures are $[\{a, b, c\},\{d\}],[\{b, c, d\},\{a\}],[\{a, c, d\},\{b\}],[\{a, b, d\},\{c\}]$, and $[N]$. We can show that

$$
M B(V ;\{a, b, c\},\{d\})=\left\{x \in \mathbb{R}_{+}^{N} \mid\left(x_{a}, x_{b}, x_{c}, x_{d}\right) \leq(1,1,1,0.5)\right\} .\left(^{*}\right)
$$

(We give a proof in the appendix.) The point $(1.5,1.5,1.5,1.5) \in V^{*}(N)$ strictly dominates in all components any payoff vector in $M B(V ;\{a, b, c\},\{d\})$, so that no profile is efficient. By symmetry, there is no efficient profile either in $M B(V ;\{b, c, d\},\{a\}), M B(V ;\{a, c, d\},\{b\})$, or $M B(V ;\{a, b, d\},\{c\})$. We can also show that

$$
M B(V ;\{a, b, c\},\{d\})=\left\{x \in \mathbb{R}_{+}^{N} \mid\left(x_{a}, x_{b}, x_{c}, x_{d}\right) \leq(1,1,1,1)\right\}
$$


Hence, there is no efficient profile in $M B(V ; N)$. Furthermore, given any payoff profile in all the above Mas-Colell bargaining sets, $(N,(1.5,1.5,1.5,1.5))$ is an objection that has no proper counterobjection. For every coalition structure $\mathcal{P}, Z B(V ; \mathcal{P}) \subset M B(V ; \mathcal{P})$, and for every efficient coalition structure, $Z B(V ; \mathcal{P})=\emptyset$. Let $x^{\prime}$ be an individually rational payoff vector in a coalition structure that is not efficient. Such a payoff profile exists uniquely: $x^{\prime}=(0.5,0.5,0.5,0.5)$. Then, $(N,(1.5,1.5,1.5,1.5))$ is an objection to $x^{\prime}$ that has no proper counterobjection. Hence, there is no coalition structure such that the Zhou bargaining set of $V$ is nonempty.

The above game is 0 -comprehensive. We can perturb it to construct a strictly 0 -comprehensive game giving rise to the same results. One would think that the improvement notion in a counterobjection is rather weak. We now consider counterobjections that improve the payoff of every member of the counterobjecting coalitions, and define the class of counterobjections that pass this strict improvement test as well as the two we have already examined. We propose the associated version of the bargaining set, which is contained in all of the other versions discussed in the paper.

Strict counterobjection: Let $V$ be a game, $x$ a payoff profile, $(S, y)$ an objection to $x$, and $T$ a coalition. Then a counterobjection $(T, z)$ to $(S, y)$ at $x$ is strict if $(T, z)$ strictly improves upon $(S, y)$ at $x$.

Strict dominating coalition: Let $V$ be a game, $x$ a payoff profile, and $S, T$ coalitions.. Then $T$ is strictly dominates $S$ at $x$ if for each objection $(S, y)$ to $x$, there is a proper and strict counterobjection $(T, z)$ to $(S, y)$ at $x$.

The Strong Steady Bargaining Set: The strong steady bargaining set of a game V for coalition structure $\mathcal{P}$ is:

$\operatorname{SSB}(V, \mathcal{P})=\{x \in I(V ; \mathcal{P}) \mid$ For each objection to $x$, there is a strictly dominant coalition at $x\}$. The strong steady bargaining set of a game $V$ is: $\operatorname{SSB}(V)=\mathrm{U}_{\mathcal{P} \in \Pi} S S B(V ; \mathcal{P})$

Given a payoff vector $x$, the inclusion relation among the sets of objections $O(x)$, objections with no counterobjections $M(x)$, those with no proper counterobjections $Z(x)$, those with no proper counterobjections $S(x)$, and those with no proper and strict counterobjections $S S(x)$ satisfy the following inclusion relations: $O(x) \supset S S(x) \supset S(x) \supset Z(x) \supset M(x)$. Then for every game $V$, we deduce the following inclusion relations among the core, the Mas-Colell bargaining set, the Zhou bargaining set, the steady bargaining set, and the strong steady bargaining set: $C_{0}(V ; \mathcal{P}) \subset$ $S S B(V ; \mathcal{P}) \subset S B(V ; \mathcal{P}) \subset Z B(V ; \mathcal{P}) \subset M B(V ; \mathcal{P})$ for every coalition structure $\mathcal{P}$, and $C_{0}(V) \subset$ $S S B(V) \subset S B(V) \subset Z B(V) \subset M B(V)$. We establish a positive result for $S S B(V)$ by assuming strict $\underline{x}$-comprehensiveness additionally.

Theorem 2: Given a game satisfying the restrictive non-crossing condition and strict $\underline{x}-$ comprehensiveness, the strong steady bargaining set contains at least one efficient payoff profile for at least one coalition structure. 
In the appendix, we give a proof of Theorem 2 that immediately follows from the proof of Theorem 1. Zhou (1994, p.520) argues that the definition of counterobjections by weak inequalities may be the "price" we have to pay in order to obtain the nonemptiness of a bargaining set for every TU game. Theorem 2 shows that this price is unnecessary as long as we assume the two properties all TU games satisfy of restrictive non-crossing condition and strict $\underline{x}$-comprehensiveness

\section{The Natural Bargaining Set and the Precise Bargaining Set}

One might feel that the proper intersection condition is too restrictive. In this section, we consider counterobjections defined by strict inequalities and weaker intersection conditions than the proper intersection condition. We define two associated versions of the bargaining set, the "natural bargaining set" and the "precise bargaining set." Their nonemptiness can be demonstrated under essentially no assumptions. We start with the weakest intersection condition to define the following type of counterobjections and the associated bargaining set:

Natural counterobjection: Let $V$ be a game, $x$ a payoff profile, $(S, y)$ an objection to $x$. Then a counterobjection $(T, z)$ to $(S, y)$ at $x$ is natural if $T \cap S \neq \varnothing$.

The Natural Bargaining Set: The natural bargaining set of a game V for coalition structure $\mathcal{P}$ is: $N B(V ; \mathcal{P})$

$=\{x \in I(V ; \mathcal{P}) \mid$ For each objection to $x$, there is a natural and strict counterobjection at $x\}$

The natural bargaining set of $V$ is: $N B(V)=\bigcup_{\mathcal{P} \in \Pi} N B(V ; \mathcal{P})$.
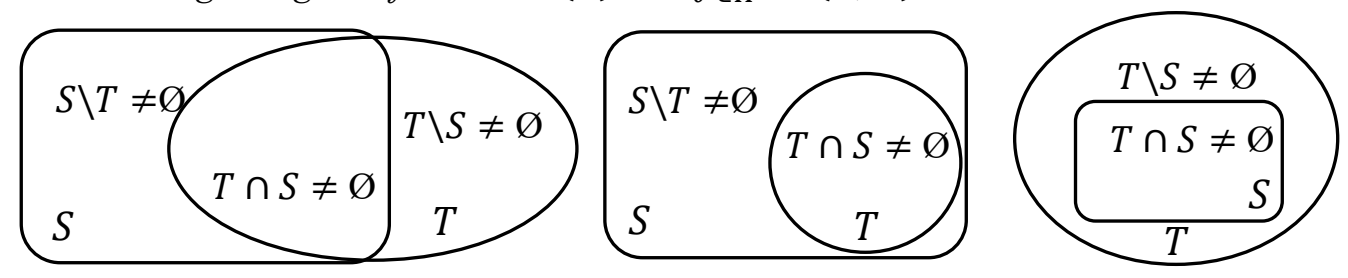

Figure 4: Coalition $S$ making an objection and coalition $T$ making a natural counterobjection

Figure 4 illustrates the possible configurations of pairs of a coalition $S$ making an objection and a coalition $T$ making a natural counterobjection. We next propose a stronger version of the bargaining set by imposing a restriction on the class of natural counterobjections:

Precise counterobjection: Let $V$ be a game, $x$ a payoff profile, $(S, y)$ an objection to $x$. Then a counterobjection $(T, z)$ to $(S, y)$ at $x$ is precise if $T \cap S \neq \varnothing$ and $S \backslash T \neq \varnothing$.

The Precise Bargaining Set: The precise bargaining set of a game V for coalition structure $\mathcal{P}$ is: $P B(V ; \mathcal{P})$

$=\{x \in I(V ; \mathcal{P}) \mid$ For each objection to $x$, there is a precise and strict counterobjection at $x\}$. The precise bargaining set of $V$ is: $P B(V)=\bigcup_{\mathcal{P} \in \Pi} P B(V ; \mathcal{P})$. 


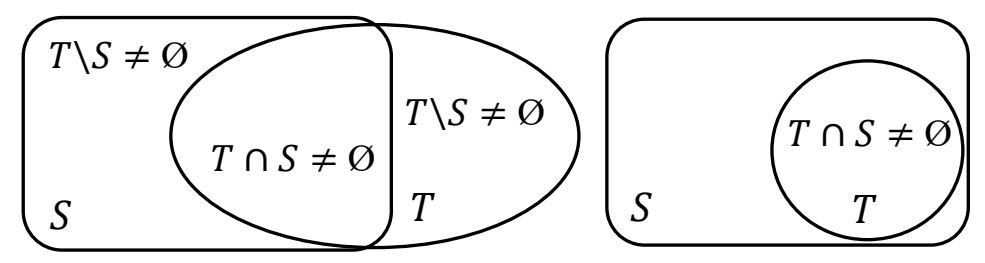

Figure 5: Coalition $S$ making an objection and coalition $T$ making a precise counterobjection

Figure 5 represents the possible configurations of coalitions making an objection and a precise counterobjection. The reason we impose the above two intersection conditions is that counterobjections to $(S, y)$ at $x$ satisfying them coincide with objections to $y$ in the reduced game on $S$ at $x$. This is a generalization of the definition of a reduced game in the TU case proposed by Aumann and Drèze (1974) in a similar way that Peleg (1985) generalized the definition of a reduced game introduced by Davis and Maschler (1965). The formulation is as follows: let $V$ be a game, $S$ a coalition, and $x$ a payoff profile. The reduced game of $V$ on $S$ at $x$ is the game $V^{x_{N \backslash S}}$ defined by

$$
V_{S}^{x_{N \backslash S}}=V_{S} \text {, and } V_{P}^{x_{N \backslash S}}=\bigcup_{Q \subset N \backslash S}\left\{y_{P} \mid\left(y_{P}, x_{Q}\right) \in V_{P \cup Q}\right\} \text { if } P \subset S \text { and } P \neq S .{ }^{5}
$$

We can see that $(S, y)$ is an objection to $x$ with no precise counterobjections if there is no objection dominating $y$ in $V_{S}^{x_{N \backslash S}}$, that is, $y$ is in the core of the reduced game. The converse is true if the possibility of improvement over $\left(y_{P}, x_{Q}\right)$ implies strict improvement (consider the case where $x \gg$ $\underline{x}$ and $V$ satisfies strict $\underline{x}$-comprehensiveness). In general, the precise bargaining set is strictly included in the natural bargaining set. See the example below.

Example 3: Two Chinese Cooks and Two Japanese Cooks. There are four cooks. Two are Chinese, and the others are Japanese: Guang, Huan, Ichiro, and Jiro. We denote them by $g, h, i$, and $j$. The problem is how to run a restaurant. Neither a single individual nor a pair of cooks from different countries can make a complete meal. Their capabilities are measured in terms of money. Let $N=$ $\{g, h, i, j\}$. The TU game $V$ describing their options is as follows: $v(\{g\})=v(\{h\})=v(\{i\})=$ $v(\{j\})=v(\{g, i\})=v(\{g, j\})=v(\{h, i\})=v(\{h, j\})=0, v(\{g, h\})=2.3, v(\{g, h, i\})=$ $v(\{g, h, j\})=3.5, v(\{i, j\})=1.3, v(\{g, i, j\})=v(\{h, i, j\})=2.5$, and $v(N)=4$, where $v(S)$ is the worth of coalition $S$. Consider the payoff profile $x=\left(x_{g}, x_{h}, x_{i}, x_{j}\right)=(1,1,1,1)$. The coalitions having objections are $\{g, h\},\{g, h, i\}$, and $\{g, h, j\}$. Note that $\{g, h\} \subset\{g, h, i\}$ and $\{g, h\} \subset\{g, h, j\}$. We can see that $x$ is in $N B(V ;\{N\})$, but not in $P B(V ;\{N\})$.

Given a payoff profile $x$, the inclusion relation among the sets of objections $O(x)$, those with no counterobjections $M(x)$, those with no proper and strict counterobjections $S S(x)$, those with no natural and strict counterobjections $N(x)$, and those with no precise objections $P(x)$ is as follows:

\footnotetext{
${ }^{5}$ This is the reduced game in the NTU case proposed by Peleg (1985). For details about reduced games in both the TU case and the NTU case, see Thomson (2021, forthcoming).
} 
$O(x) \supset S S(x) \supset P(x) \supset N(x) \supset M(x)$. Then for every game $V$, we deduce the following inclusion relations among the core, the Mas-Colell Bargaining Set, the strong steady bargaining set, the natural bargaining set, and the precise bargaining Set: $C_{0}(V ; \mathcal{P}) \subset S S B(V ; \mathcal{P}) \subset P B(V ; \mathcal{P}) \subset N B(V ; \mathcal{P}) \subset$ $M B(V ; \mathcal{P})$ for every coalition structure $\mathcal{P}$, and $C_{0}(V) \subset S S B(V) \subset P B(V) \subset N B(V) \subset M B(V)$.

Neither the natural bargaining set nor the precise bargaining set contains the steady bargaining set. The counterobjections to determine the steady bargaining set satisfy a stronger intersection condition than that of the precise bargaining set, but they are not defined by strict inequalities. The intersection requirments make the natural, and the precise, bargaining sets larger, and the strict inequalities make them smaller than the steady bargaining set.

The following theorem tells us that the above two refinements of the Mas-Colell bargaining set are nonvacuous in every coalitional game.

Theorem 3: The Precise bargaining set of a game contains at least one payoff profile for at least one coalition structure.

Corollary: The Mas-Colell Bargaining Set and the Natural Bargaining Set of a game contain at least one payoff profile for at least one coalition structure.

As long as $V_{S} \cap\left(\left\{r_{S}\right\}+\mathbb{R}_{+}^{S}\right)$ is compact for every coalition $S$, we do not need all four conditions of a coalitional game to establish the result. Notice that Theorem 3 does not say anything about efficiency, and recall Example 2; the Mas-Colell bargaining set may not contain any of the efficient profiles if the game does not satisfy the non-crossing condition. Thus, there may be no efficient profile in $P B(V)$ or $N B(V)$.

\section{Concluding Remarks}

In cooperative game theory, some solutions describe stable compromises in noncooperative situations among coalitions [see Mas-Colell (1987), and Vohra (1995) for surveys]. Although there are several studies of the bargaining set of NTU games [see Iñarra et al. (2020), p.260], little research has been done on the bargaining set of NTU games with coalition structures. Our model, as well as Zhou's (1994), describes coalition formation as an outcome of the bargaining set. By applying the bargaining set, we have explained how coalition formations and payoff allocations are simultaneously determined in coalitional NTU games. The theory of the core successfully explains coalition formation in limited classes of NTU games, such as one-to-one matchings. Even though the core is empty for many problems, other classical solutions work for coalitional games. Hence, there is no doubt that cooperative solutions will continue to be useful in considering multi-person coalition formation together with payoff allocation in various contexts. 


\section{Appendix}

Proof of Theorem 1. We use a fixed-point argument. The key to the proof is the construction of the fixed-point correspondence. In particular, the homeomorphism $h$, defined in Step 1, plays a crucial role. The existence of $h$ heavily depends on where $\underline{x}$ is, namely the conditions of $\underline{x}$ interiority and $\underline{x}$-comprehensiveness.

$\underline{\text { Step } 1}$. We define functions $f^{S}$ for $S \subset N$ to use for construction of the fixed-point correspondence. Let $\mathcal{N}$ be the class of coalitions. For every coalition $S$ with $1<|S|<|N|$, define $\mathcal{T}(S) \equiv\{T \in \mathcal{N} \mid$ $T \cap S \neq \emptyset \& S \backslash T \neq \varnothing \& T \backslash S \neq \emptyset\}$. There is $\underline{x} \in \mathbb{R}^{N}$ such that $V$ satisfies $\underline{x}$-compactness, $\underline{x}$ interiority and $\underline{x}$-comprehensiveness. By $\underline{x}$-interiority, $\underline{x} \in \bigcap_{i \in N} \operatorname{int} V(\{i\})$. Then $\underline{x} \in \operatorname{int} V^{*}$. By $\underline{x}$ compactness, $\bar{V}_{S} \cap\left[\left\{\underline{x}_{S}\right\}+\mathbb{R}_{+}^{S}\right]$ is compact for every $S \in \mathcal{N}$. Hence, $V_{+}^{*} \equiv V^{*} \cap\left[\{\underline{x}\}+\mathbb{R}_{+}^{N}\right]$ is compact. Let $Z \equiv\left\{z \in \mathbb{R}_{+}^{N} \mid z \cdot e \leq 1\right\}$ and $\Delta \equiv\left\{z \in \mathbb{R}_{+}^{N} \mid z \cdot e=1\right\}$, where $e=(1, \ldots, 1)$.

Define the function $h: Z \rightarrow V_{+}^{*}$ by

$$
h(z) \equiv \begin{cases}\underline{x} & \text { if } z=0 \\ \underline{x}+c(z /(z \cdot e)) z & \text { if } z \neq 0\end{cases}
$$

for all $z \in Z$, where $c(y) \equiv\left\{t \in \mathbb{R}_{+} \mid \underline{x}+t y \in P\left(V^{*}\right)\right\}$ for every $y \in \Delta$. By $\underline{x}$-interiority and $\underline{x}$ comprehensiveness, $c(z /(z \cdot e))$ uniquely exists for each $z \in Z \backslash\{0\}$ and $h$ is a homeomorphism from $Z$ onto $V_{+}^{*}$ (See Figures 6). For every $(z, i) \in Z \times N$, note that $z_{i}=0$ if and only if $h_{i}(z)=\underline{x}_{i}$. In addition, $h(z) \in P\left(V^{*}\right)$ if $z \in \Delta$.

Let $S \in \mathcal{N}$. By continuity, there is a continuous representation $v^{S}: \mathbb{R}^{N} \rightarrow \mathbb{R}$ for $V_{S}$. Let $i \in N$. By $\underline{x}$-compactness, $\max V_{\{i\}}$ exists. Define $r \in \mathbb{R}^{N}$ by $r_{i} \equiv \max V_{\{i\}}$ for all $i \in N$. For every $x_{S} \in \mathbb{R}^{S}$, define $\left[x_{S}\right] \equiv\left\{z_{S} \in \mathbb{R}^{S} \mid z_{i}=\max \left\{x_{i}, r_{i}\right\}\right.$ for each $\left.i \in S\right\}$.

For all $S \in \mathcal{N}$, define $f^{S}: Z \rightarrow \mathbb{R}_{+}$as follows: If $|S|=1$,or $|N|$, then

$$
f^{S}(z) \equiv \max \left\{-v^{S}\left(h_{S}(z)\right), 0\right\}
$$

for every $z \in Z$. If $1<|S|<|N|$, let $D^{S} \equiv\left\{z \in Z \mid v^{S}\left(\left[h_{S}(z)\right]\right)<0\right\}$, and define

$$
\begin{aligned}
& O_{T}^{S}(z) \equiv\left\{y_{S \cap T} \in\left\{\left[h_{S \cap T}(z)\right]\right\}+\mathbb{R}_{++}^{S \cap T} \mid v^{S}\left(y_{S \cap T}, h_{S \backslash T}(z)\right)<0\right\}, \text { where } T \in \mathcal{T}(S), \\
& m^{T}(z) \equiv \max \left\{v^{T}\left(y_{S \cap T}, h_{S \backslash T}(z)\right) \mid y_{S \cap T} \in \operatorname{cl} O_{T}^{S}(z)\right\}, \text { where } T \in \mathcal{T}(S) \text {, and } \\
& e^{S}(z) \equiv \min \left\{m^{T}(z) \mid T \in \mathcal{T}(S)\right\}
\end{aligned}
$$

where $\operatorname{cl} O_{T}^{S}(z)$ is the closure of $O_{T}^{S}(z)$, for every $z \in D^{S}$. Finally, define

$$
f^{S}(z) \equiv\left\{\begin{array}{cl}
\max \left\{-v^{S}\left(\left[h_{S}(z)\right]\right), 0\right\} \max \left\{e^{S}(z), 0\right\} & \text { if } z \in D^{S} \\
\max \left\{-v^{S}\left(h_{S}(z)\right), 0\right\} & \text { if } z \in Z \backslash D^{S}
\end{array}\right.
$$

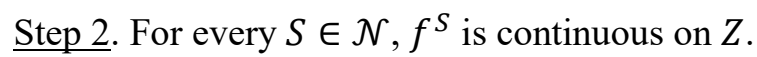

Proof of Step 2. Let $S \in \mathcal{N}$ be with $|S|=1$, or $|N|$. Since $v^{S}$ is continuous, so is $f^{S}$.

Next, fix $S \subset N$ such that $1<|S|<|N|$. Let $T \in \mathcal{T}(S)$ and $z \in D^{S}$. Since $v^{S}\left(\left[h_{S}(z)\right]\right)<0$ and

$\left[h_{S \backslash T}(z)\right] \geq h_{S \backslash T}(z), \quad \underline{x}$-compehensiveness implies $v^{S}\left(\left[h_{S \cap T}(z)\right], h_{S \backslash T}(z)\right)<0$. Since $v^{S}$ is continuous, there exist $y_{S \cap T} \in\left\{\left[h_{S \cap T}(z)\right]\right\}+\mathbb{R}_{++}^{S \cap T}$ such that $v^{S}\left(y_{S \cap T}, h_{S \backslash T}(z)\right)<0$. Thus, $O_{T}^{S}(z) \neq \varnothing$. Then, $\operatorname{cl} O_{T}^{S}$ is the correspondence from $D^{S}$ to $\mathbb{R}^{S \cap T}$ that assigns to each $z \in D^{S}$ the closure of $O_{T}^{S}(z)$. 


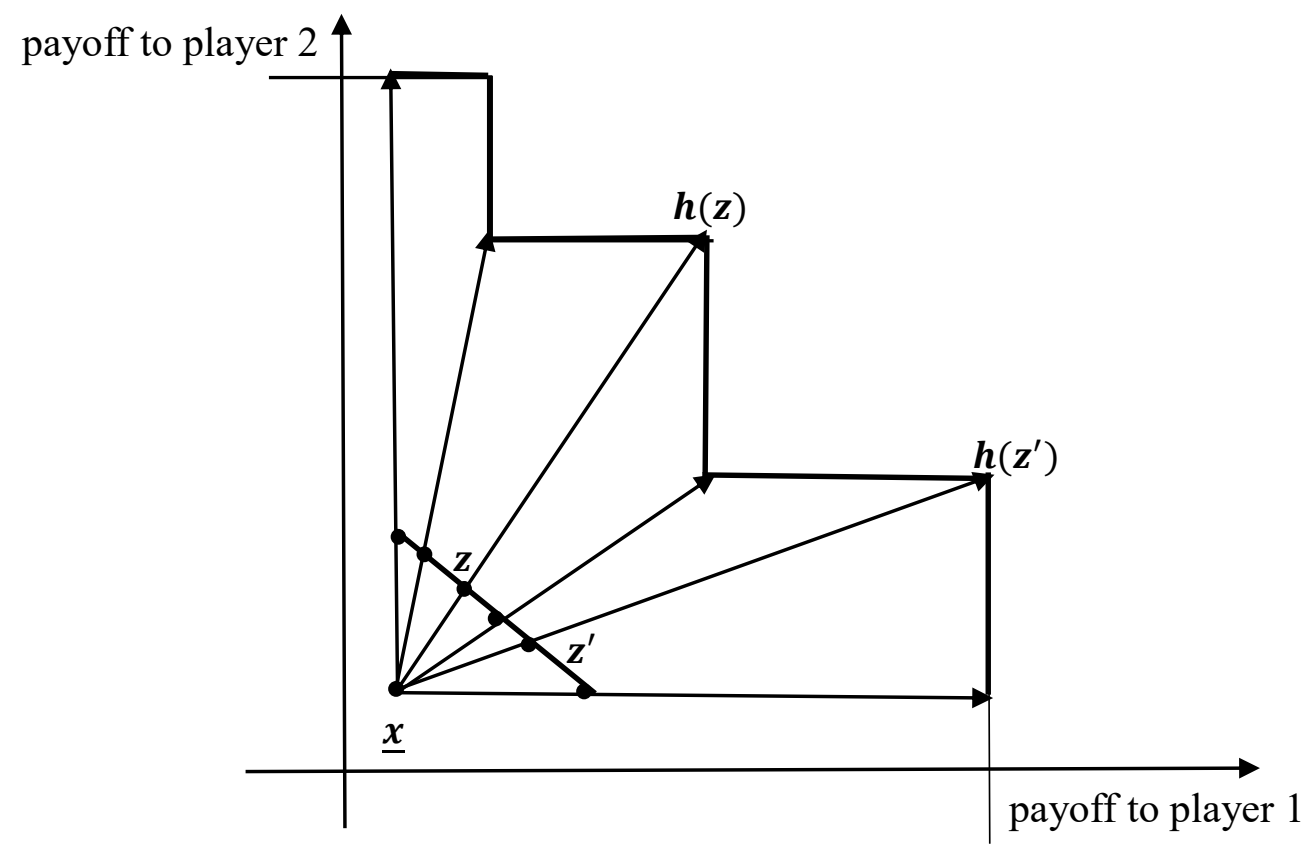

payoff to player 3

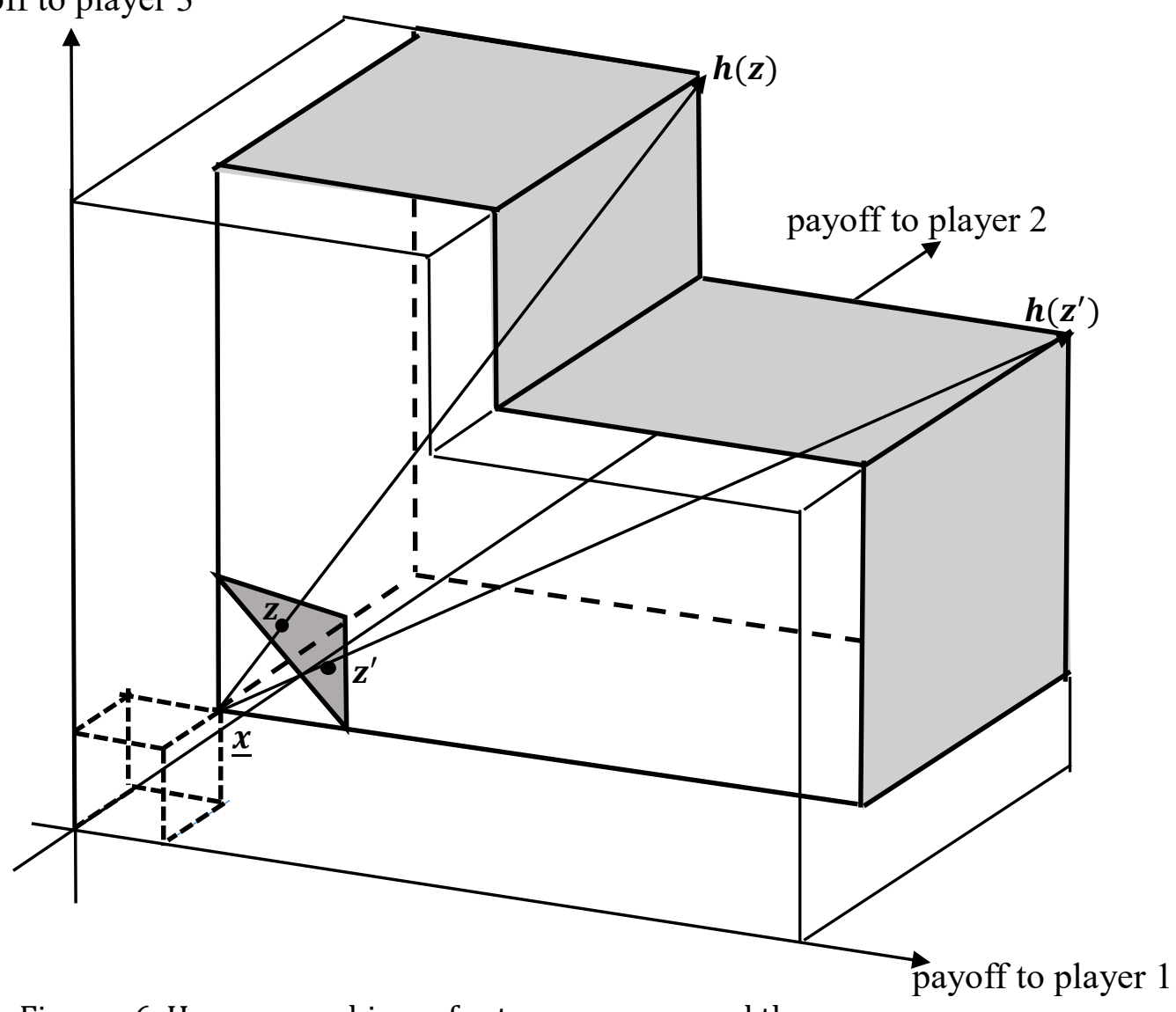

Figures 6: Homeomorphisms for two - person and three - person games 
We show that the correspondence $\operatorname{cl} O_{T}^{S}$ is compact-valued, upper-hemicontinuous, and lowerhemicontinuous on $D^{S}$. Since $V_{S} \cap\left[\left\{\underline{x}_{S}\right\}+\mathbb{R}_{+}^{S}\right]$ is compact and $h_{S}(z) \geq \underline{x}_{S}$, clO $O_{T}^{S}$ is compactvalued. Since $Z$ is compact and $h$ is continuous, $h_{S \backslash T}(Z)$ is compact, so that the range of $\operatorname{cl} O_{T}^{S}$ is included in a compact subset of $\mathbb{R}^{S \cap T}$.

To show the upper-hemicontinuity of $\operatorname{cl} O_{T}^{S}$, we prove that the graph of $\operatorname{cl} O_{T}^{S}$ is closed in $D^{S} \times \mathbb{R}^{S \cap T}$. Let $\left(z, y_{S \cap T}\right) \in D^{S} \times \mathbb{R}^{S \cap T}$ and $\left\{\left(z^{q}, y_{S \cap T}^{q}\right)\right\}$ be a sequence in $D^{S} \times \mathbb{R}^{S \cap T}$ such that $y_{S \cap T}^{q} \in \operatorname{cl} O_{T}^{S}\left(z^{q}\right)$ and $\left(z^{q}, y_{S \cap T}^{q}\right) \rightarrow\left(z, y_{S \cap T}\right)$. We show $y_{S \cap T} \in \operatorname{cl} O_{T}^{S}(z)$. For each $\varepsilon>0$ and every $x_{S \cap T} \in \mathbb{R}^{S \cap T}$, define $B\left(\varepsilon ; x_{S \cap T}\right) \equiv\left\{y_{S \cap T} \in \mathbb{R}^{S \cap T} \mid\left\|y_{S \cap T}-x_{S \cap T}\right\|<\varepsilon\right\}$ (the open ball with $x_{S \cap T}$ center and $\varepsilon$ radius). Since $y_{S \cap T}^{q} \rightarrow y_{S \cap T}$, there is a subsequence $\{q(k)\}$ of $\{q\}$ such that

$$
\forall k \in \mathbb{N}, \forall q \in \mathbb{N}\left[q \geq q(k) \Rightarrow y_{S \cap T}^{q} \in B\left(1 / k ; y_{S \cap T}\right)\right]
$$

Since $y_{S \cap T}^{q(k)} \in \operatorname{cl} O_{T}^{S}\left(z^{q(k)}\right)$, it follows that

$B\left(1 / k ; y_{S \cap T}\right) \cap\left(\left\{\left[h_{S \cap T}\left(z^{q(k)}\right)\right]\right\}+\mathbb{R}_{++}^{S \cap T}\right) \cap\left\{y_{S \cap T} \in \mathbb{R}^{S \cap T} \mid v^{S}\left(y_{S \cap T}, h_{S \backslash T}\left(z^{q(k)}\right)\right)<0\right\} \neq \emptyset$.

Take

$$
y_{S \cap T}^{k} \in B\left(1 / k ; y_{S \cap T}\right) \cap\left(\left\{\left[h_{S \cap T}\left(z^{q(k)}\right)\right]\right\}+\mathbb{R}_{++}^{S \cap T}\right) \cap\left\{y_{S \cap T} \in \mathbb{R}^{S \cap T} \mid v^{S}\left(y_{S \cap T}, h_{S \backslash T}\left(z^{q(k)}\right)\right)<0\right\}
$$

Then, $y_{S \cap T}^{k} \in B\left(1 / k ; y_{S \cap T}\right)$, and $y_{S \cap T}^{k} \rightarrow y_{S \cap T}$. Since $z^{q} \rightarrow z$, we have $z^{q(k)} \rightarrow z$. In addition, $y_{S \cap T}^{k} \in\left(\left\{\left[h_{S \cap T}\left(z^{q(k)}\right)\right]\right\}+\mathbb{R}_{++}^{S \cap T}\right) \cap\left\{y_{S \cap T} \in \mathbb{R}^{S \cap T} \mid v^{S}\left(y_{S \cap T}, h_{S \backslash T}\left(z^{q(k)}\right)\right)<0\right\}$. $\quad$ Since $y_{S \cap T}^{k} \gg\left[h_{S \cap T}\left(z^{q(k)}\right)\right]$, it follows by the continuity of $h_{S \cap T}$ that $y_{S \cap T} \geq\left[h_{S \cap T}(z)\right]$. Note that $v^{S}\left(y_{S \cap T}, h_{S \backslash T}\left(z^{q(k)}\right)\right)<0$. By the continuity of $h_{S \backslash T}$ and $v^{S}, \quad v^{S}\left(y_{S \cap T}, h_{S \backslash T}(z)\right) \leq 0$. Thus, $y_{S \cap T} \in \operatorname{cl} O_{T}^{S}(z)$. Hence $\operatorname{cl} O_{T}^{S}$ has a closed graph, so that $\operatorname{cl} O_{T}^{S}$ is upper-hemicontinuous.

We next show that $\operatorname{cl} O_{T}^{S}$ is lower-hemicontinuous on $D^{S}$. Let $z \in D^{S}$, and $U$ be an open subset of $\mathbb{R}^{S \cap T}$ such that $O_{T}^{S}(z) \cap U \neq \varnothing$. Then there is $y_{S \cap T} \in U$ such that $y_{S \cap T} \gg\left[h_{S \cap T}(z)\right]$ and $v^{S}\left(y_{S \cap T}, h_{S \backslash T}(z)\right)<0$. Since $h$ is continuous, there is an open neighborhood $W$ of $z$ such that $y_{S \cap T} \gg\left[h_{S \cap T}\left(z^{\prime}\right)\right]$ and $v^{S}\left(y_{S \cap T}, h_{S \backslash T}\left(z^{\prime}\right)\right)<0$ for all $z^{\prime} \in W$. Hence, $y_{S \cap T} \in O_{T}^{S}\left(z^{\prime}\right) \cap U$, namely $O_{T}^{S}\left(z^{\prime}\right) \cap U \neq \varnothing$ for every $z^{\prime} \in W$. Thus, $O_{T}^{S}$ is lower-hemicontinuous, and so is $\operatorname{cl} O_{T}^{S}$.

Recall $m^{T}(z)=\max \left\{v^{T}\left(y_{S \cap T}, h_{S \backslash T}(z)\right) \mid y_{S \cap T} \in \operatorname{cl} O_{T}^{S}(z)\right\}$. Since $v^{T}$ is continuous, by the Berge maximum theorem, $m^{T}$ is continuous. Recall $e^{S}(z) \equiv \min \left\{m^{T}(z) \mid T \in \mathcal{T}(S)\right\}$, then $e^{S}$ is also continuous. Since there is a compact subset of $\mathbb{R}^{S}$ including the range of $\operatorname{cl} O_{T}^{S}$ and $\mathcal{T}(S)$ is finite, $e^{S}$ has an upper bound.

We finally show that $f^{S}$ is continuous on $Z$. Let $z \in D^{S}$, then $e^{S}$ and $f^{S}$ is continuous at $z$. Next choose $z^{*} \in Z \backslash D^{S}$, then $v^{S}\left(\left[h_{S}\left(z^{*}\right)\right]\right) \geq 0$ and $f^{S}(z)=\max \left\{-v^{S}\left(\left[h_{S}\left(z^{*}\right)\right]\right), 0\right\}=0$. Let $\left\{z^{q}\right\}$ be a sequence in $Z$ such that $z^{q} \rightarrow z^{*}$, then $\max \left\{-v^{S}\left(\left[h_{S}\left(z^{q}\right)\right]\right), 0\right\} \rightarrow 0$. Since $e^{S}$ has an upper bound, $\max \left\{-v^{S}\left(\left[h_{S}\left(z^{q}\right)\right]\right), 0\right\} \max \left\{e^{S}\left(z^{q}\right), 0\right\} \rightarrow 0$, so that $f^{S}\left(z^{q}\right) \rightarrow 0$. Hence, $f^{S}$ is continuous at $z^{*}$. Thus, $f^{S}$ is continuous on $Z$.

$\underline{\text { Step 3 }}$. We construct the fixed-point correspondence $\varphi$ by $f^{S}$, and apply the Kakutani theorem. Define the function $F: Z \rightarrow \mathbb{R}_{+}^{N}$ by 


$$
F_{i}(z) \equiv \max \left\{f^{S}(z) \mid S \in \mathcal{N} \text { with } i \in S\right\}
$$

for every $z \in Z$, then $F$ is continuous. Define the correspondence $\varphi: \Delta \rightarrow \Delta$ by

$$
\varphi(z) \equiv\left\{z^{*} \in \Delta \mid\left(\forall z^{\prime} \in \Delta\right)\left(z^{*} \cdot F(z) \geq z^{\prime} \cdot F(z)\right)\right\}
$$

for every $z \in \Delta$. Note that $\varphi(z)=\Delta$ if $F(z)=0$. By the Berge maximum theorem, $\varphi$ is compactvalued, and upper-hemicontinuous. Further, $\varphi$ is convex-valued. By the Kakutani fixed-point theorem [Kakutani (1941)], there exists $z^{*} \in \Delta$ such that $z^{*} \in \varphi\left(z^{*}\right)$. That is, $z^{*} \cdot F\left(z^{*}\right) \geq z$. $F\left(z^{*}\right)$ for all $z \in \Delta$.

Step 4. The payoff profile $x^{*}$ associated with a fixed-point is efficient.

Proof of Step 4.Let $x^{*}=h\left(z^{*}\right)$. Since $z^{*} \in \Delta, x^{*} \in P\left(V^{*}\right)$. Thus, $x^{*}$ is an efficient payoff profile.

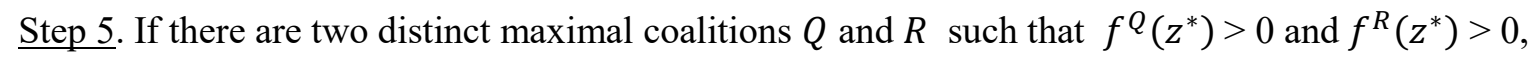
then they are disjoint, namely $Q \cap R=\varnothing$.

Proof of Step 5. Let $Q, R \in \mathcal{N}$ be two distinct maximal coalitions such that $f^{Q}\left(z^{*}\right)>0$ and $f^{R}\left(z^{*}\right)>$ 0 . We show that $Q \cap R=\varnothing$. We must have that $|Q|<|N|$, and $|R|<|N|$. If $|Q|=1$ or $|R|=1$, then it follows by maximality that $Q \cap R=\varnothing$. If $|Q|>1$ and $|R|>1$, then

$$
\begin{aligned}
& v^{Q}\left(\left[x_{Q}^{*}\right]\right)<0 \&(\forall T \in \mathcal{T}(Q))\left(\exists y_{Q} \in \operatorname{cl} O_{T}^{Q}\left(z^{*}\right)\right)\left(v^{T}\left(y_{T \cap Q}, x_{T \backslash Q}^{*}\right)>0\right) \\
& v^{R}\left(\left[x_{R}^{*}\right]\right)<0 \&(\forall T \in \mathcal{T}(R))\left(\exists y_{R}^{\prime} \in \operatorname{cl} O_{T}^{Q}\left(z^{*}\right)\right)\left(v^{T}\left(y_{T \cap R}^{\prime}, x_{T \backslash R}^{*}\right)>0\right) .
\end{aligned}
$$

Suppose that $Q \cap R \neq \varnothing$. By maximality, $Q \backslash R \neq \varnothing$ and $R \backslash Q \neq \varnothing$, so that $R \in \mathcal{T}(Q)$ and $Q \in$ $\mathcal{T}(R)$. Further, recall the definition of $O_{T}^{S}(z)$. By continuity, we may assume that

$$
\left(\exists y_{Q} \in O_{R}^{Q}\left(z^{*}\right)\right)\left(v^{R}\left(y_{Q \cap R}, x_{R \backslash Q}^{*}\right)>0\right) \&\left(\exists y_{R}^{\prime} \in \operatorname{cl} O_{Q}^{R}\left(z^{*}\right)\right)\left(v^{Q}\left(y_{Q \cap R}^{\prime}, x_{Q \backslash R}^{*}\right)>0\right) .
$$

Note that $y_{Q} \in O_{R}^{Q}\left(z^{*}\right)$ means $y_{Q} \gg\left[h_{Q}\left(z^{*}\right)\right]=x_{Q}^{*}$ and $v^{Q}\left(y_{Q \cap R}, x_{Q \backslash R}^{*}\right)<0$. Similarly, $y_{R}^{\prime} \in$ $\operatorname{cl} O_{Q}^{R}\left(z^{*}\right)$ means $y_{R}{ }^{\prime} \gg\left[h_{R}\left(z^{*}\right)\right]=x_{R}^{*}$ and $v^{R}\left(y_{Q \cap R}, x_{R \backslash Q}^{*}\right)<0$. Then

$$
\begin{aligned}
& {\left[x_{Q \cap R}^{*}\right] \ll y_{Q \cap R} \& v^{Q}\left(y_{Q \cap R}, x_{Q \backslash R}^{*}\right)<0 \& v^{R}\left(y_{Q \cap R}, x_{R \backslash Q}^{*}\right)>0} \\
& {\left[x_{Q \cap R}^{*}\right] \ll y_{Q \cap R}^{\prime} \& v^{R}\left(y_{Q \cap R}^{\prime}, x_{R \backslash Q}^{*}\right)<0 \& v^{Q}\left(y_{Q \cap R}^{\prime}, x_{Q \backslash R}^{*}\right)>0}
\end{aligned}
$$

Hence,

$$
\begin{gathered}
v^{Q}\left(y_{Q \cap R}, x_{Q \backslash R}^{*}\right)<0<v^{Q}\left(y_{Q \cap R}^{\prime}, x_{Q \backslash R}^{*}\right) \& v^{R}\left(y_{Q \cap R}^{\prime}, x_{R \backslash Q}^{*}\right)<0<v^{R}\left(y_{Q \cap R}^{\prime}, x_{R \backslash Q}^{*}\right), \\
\text { i.e., }\left(y_{Q \cap R}, x_{Q \backslash R}^{*}\right) \in \operatorname{int} V_{Q} \&\left(y_{Q \cap R}^{\prime}, x_{Q \backslash R}^{*}\right) \notin V_{Q} \&\left(y_{Q \cap R}^{\prime}, x_{R \backslash Q}^{*}\right) \in \operatorname{int} V_{R} \&\left(y_{Q \cap R}^{\prime}, x_{R \backslash Q}^{*}\right) \notin V_{R}
\end{gathered}
$$

Note that $y_{Q \cap R} \gg\left[x_{Q \cap R}^{*}\right] \geq r_{Q \cap R}$ and $y_{Q \cap R}^{\prime} \gg\left[x_{Q \cap R}^{*}\right] \geq r_{Q \cap R}$. Since $x^{*} \in P\left(V^{*}\right)$, this contradicts the restrictive non-crossing condition. Thus, $Q \cap R=\varnothing$.

Step 6. For all $S \in \mathcal{N}, f^{S}\left(z^{*}\right)=0$.

Proof of Step 6. The proof is by contradiction. Suppose there is $S \in \mathcal{N}$ with $f^{S}\left(z^{*}\right)>0$.

We first prove that there is a partition $\mathcal{P}$ of $N$ such that $f^{Q}\left(z^{*}\right)>0$ for all $Q \in \mathcal{P}$ : Let $f^{S}\left(z^{*}\right)>0$, then $F\left(z^{*}\right) \geq 0$ and $F\left(z^{*}\right) \neq 0$. Let $i \in N$. If $F_{i}\left(z^{*}\right)=0$, then $0=f^{\{i\}}\left(z^{*}\right)=$ $\max \left\{-v^{\{i\}}\left(h_{i}\left(z^{*}\right)\right), 0\right\}=\max \left\{-v^{\{i\}}\left(x_{i}^{*}\right), 0\right\}$. Thus, $v^{\{i\}}\left(x_{i}^{*}\right) \geq 0$. Since $F\left(z^{*}\right) \geq 0, F\left(z^{*}\right) \neq 0$ 
and $z^{*} \in \varphi\left(z^{*}\right)$, we have $z_{i}^{*}=0$. By the construction of $h$ and $\underline{x}$-interiority, we have $x_{i}^{*}=$ $h_{i}\left(z^{*}\right)=\underline{x}_{i} \in \operatorname{int} V_{\{i\}}$. This contradicts the inequality $v^{\{i\}}\left(x_{i}^{*}\right) \geq 0$. Thus, for every $i \in N$, $F_{i}\left(z^{*}\right)>0$. Hence, for every $i \in N$, there is $S(i) \in \mathcal{N}$ such that $i \in S(i)$ and $f^{S(i)}\left(z^{*}\right)>0$. Note that $\cup_{i \in N} S(i)=N$. Then, there exist a class $\mathcal{P}$ of maximal coalitions such that $f^{Q}\left(z^{*}\right)>0$ for all $Q \in \mathcal{P}$ and $\bigcup_{Q \in \mathcal{P}} Q=N$. By Step 5, $\mathcal{P}$ is a partition of $N$.

Let $S \in \mathcal{P}$. Since $v^{S}\left(\left[x_{S}^{*}\right]\right)<0$ and $x_{S}^{*} \leq\left[x_{S}^{*}\right]$, we have $v^{S}\left(x_{S}^{*}\right)<0$, so that $x_{S}^{*} \in \operatorname{int} V_{S}$. Thus, $x_{S}^{*} \in \operatorname{int} V_{S}$ for all $S \in \mathcal{P}$. Hence, $x^{*} \in \operatorname{int} V^{*}$. Recall $x^{*} \in P\left(V^{*}\right)$. This is a contradiction. Hence, there is no $S \in \mathcal{N}$ such that $f^{S}\left(z^{*}\right)>0$. That is, all $S \in \mathcal{N}, f^{S}\left(z^{*}\right)=0$.

Step 7. $x^{*} \in S B(V)$.

Proof of Step 7. Let $i \in N$. Since $0=f^{\{i\}}\left(z^{*}\right), v^{\{i\}}\left(x_{i}^{*}\right) \geq 0$, namely $x_{i}^{*} \notin \operatorname{int} V_{\{i\}}$. Thus, $x_{i}^{*} \geq r_{i}$, and $x_{S}^{*}=\left[x_{S}^{*}\right] \gg x_{S}$ for all $S \in \mathcal{N}$. Since $0=f^{N}\left(z^{*}\right)=\max \left\{-v^{N}\left(h\left(z^{*}\right)\right), 0\right\}=\max \left\{-v^{N}\left(x^{*}\right), 0\right\}$, we also have $x^{*} \notin \operatorname{int} V_{N}$. Let $S \in \mathcal{N}$ with $1<|S|<|N|$ satisfy $0>v^{S}\left(\left[x_{S}^{*}\right]\right)=v^{S}\left(x_{S}^{*}\right)$. Then, $\max \left\{e^{S}\left(z^{*}\right), 0\right\}=0$, so that $e^{S}\left(z^{*}\right) \leq 0$. By $\underline{x}$-comprehensiveness, $y_{S \cap T} \in \operatorname{cl} O_{T}^{S}\left(z^{*}\right)$ if $T \in$ $\mathcal{T}(S), y_{S \cap T} \gg x_{S \cap T}^{*}$ and $v^{S}\left(y_{S \cap T}, x_{S \backslash T}^{*}\right) \leq 0$. By the definition of $e^{S}$, there is $T \in \mathcal{T}(S)$ such that

$$
\forall y_{S \cap T} \in\left\{x_{S \cap T}^{*}\right\}+\mathbb{R}_{++}^{S \cap T} \text { s.t. } v^{S}\left(y_{S \cap T}, x_{S \backslash T}^{*}\right) \leq 0, v^{T}\left(y_{S \cap T}, x_{T \backslash S}^{*}\right) \leq 0(* *)
$$

Let $(S, y)$ be an objection to $x^{*}$. Then $y \gg x_{S}^{*}$. Since $y_{S \cap T} \gg x_{S \cap T}^{*}, v^{T}\left(y_{S \cap T}, x_{T \backslash S}^{*}\right) \leq 0$. Thus, there is a proper counterobjection $\left(T,\left(y_{S \cap T}, x_{T \backslash S}^{*}\right)\right)$ to $(S, y)$ at $x^{*}$. Hence, $x^{*} \in S B(V)$.

This completes the proof of Theorem 1.

Proof of Theorem 2. Suppose $V$ satisfies strict $\underline{x}$-comprehensiveness. Let $x^{*},(S, y)$, and $T$ be respectively the same profile, objection, and dominant coalition to $S$ at $x^{*}$ as in the proof of Theorem 1. Recall $y=\left(y_{S \cap T}, y_{S \backslash T}\right) \geq\left(y_{S \cap T}, x_{S \backslash T}^{*}\right), y \neq\left(y_{S \cap T}, x_{S \backslash T}^{*}\right)$, and $\left(y_{S \cap T}, x_{S \backslash T}^{*}\right) \geq \underline{x}_{S}$. By strict $\underline{x}$-comprehensiveness, $\left(y_{S \cap T}, x_{S \backslash T}^{*}\right) \in \operatorname{int} V_{S}$, and there is $y^{\prime}=\left(y_{S \cap T}^{\prime}, x_{S \backslash T}^{*}\right) \in V_{S}$ such that $y_{S \cap T}^{\prime} \gg y_{S \cap T}$. Note that $y_{S \cap T}^{\prime} \gg y_{S \cap T} \gg x_{S \cap T}^{*}$ and $v^{S}\left(y_{S \cap T}, x_{S \backslash T}^{*}\right)<0$. By (**) in the proof of Theorem 1, $v^{T}\left(y_{S \cap T}^{\prime}, x_{T \backslash S}^{*}\right) \leq 0$. Note that $\left(y_{S \cap T}^{\prime}, x_{T \backslash S}^{*}\right) \geq\left(y_{S \cap T}, x_{T \backslash S}^{*}\right),\left(y_{S \cap T}^{\prime}, x_{T \backslash S}^{*}\right) \neq$ $\left(y_{S \cap T}, x_{T \backslash S}^{*}\right)$, and $\left(y_{S \cap T}, x_{T \backslash S}^{*}\right) \geq \underline{x}_{T}$. By strict $\underline{x}$-comprehensiveness, $\left(y_{S \cap T}, x_{T \backslash S}^{*}\right) \in \operatorname{int} V_{T}$, so that there is $z \in V_{T}$ such that $z \gg\left(y_{S \cap T}, x_{T \backslash S}^{*}\right)$. Thus, $(T, z)$ is a strict and proper counterobjection to $y$ at $x^{*}$, and $T$ is strictly dominant to $S$ at $x^{*}$. Hence, $x^{*} \in \operatorname{SSB}(V)$.

Proof of (*) in Example 2. We have $I(V ;\{a, b, c\},\{d\})=\left\{x \in \mathbb{R}_{+}^{N} \mid\left(x_{a}, x_{b}, x_{c}, x_{d}\right) \leq(6,4,1,0)\right\}$. Let $x^{*} \in M B(V ;\{a, b, c\},\{d\})$. Note that $M B(V ;\{a, b, c\},\{d\}) \subset I(V ;\{a, b, c\},\{d\})$. The pair $(\{b, c, d\},(6,4,1))$ is an objection to any payoff profile of $I(V ;\{a, b, c\},\{d\})$. For $x^{*}$ to be in $M B(V ;\{a, b, c\},\{d\})$, there need to be a counterobjection to $(\{b, c, d\},(6,4,1))$ at $x^{*}$. We see that $\{a, c, d\}$ is the only coalition that can make such a counterobjection if and only if $x_{a}^{*} \leq 1$. We then suppose $x_{a}^{*} \leq 1$. The pair $(\{a, c, d\},(2,3,5))$ is an objection to any payoff profile of 
$I(V ;\{a, b, c\},\{d\}) \cap\left\{x \in \mathbb{R}_{+}^{N} \mid\left(x_{a}, x_{b}, x_{c}, x_{d}\right) \leq(1,1,1,0)\right\}$. For $\quad x^{*} \in M B(V ;\{a, b, c\},\{d\})$, there need to be a counterobjection to $(\{a, c, d\},(2,3,5))$ at $x^{*}$. We see that $\{a, b, d\}$ is the only coalition that can make such a counterobjection if and only if $x_{b}^{*} \leq 1$. Hence,

$$
M B(V ;\{a, b, c\},\{d\}) \subset I(V ;\{a, b, c\},\{d\})\} .
$$

Conversely, let $x^{*} \in\left\{x \in \mathbb{R}_{+}^{N} \mid\left(x_{a}, x_{b}, x_{c}, x_{d}\right) \leq(1,1,1,0)\right\}$. Then given $x^{*}$, any objection from $\{a, b, c\}$ (resp., $\{b, c, d\},\{a, c, d\}$, and $\{a, b, d\})$ has the counterobjection $(\{b, c, d\},(6,4,1)$ ) (resp., $(\{a, c, d\},(1,6,4)),(\{a, b, d\},(4,1,6))$, and $(\{a, b, c\},(6,4,1))$. In addition, given $x^{*}$, any objection from $N$ has the counterobjection $(\{a, b, c\},(3,5,2))$. Hence, we have $\left\{x \in \mathbb{R}_{+}^{N} \mid\left(x_{a}, x_{b}, x_{c}, x_{d}\right) \leq(1,1,1,0)\right\} \subset M B(V ;\{a, b, c\},\{d\})$. It therefore follows that

$$
M B(V ;\{a, b, c\},\{d\})=\left\{x \in \mathbb{R}_{+}^{N} \mid\left(x_{a}, x_{b}, x_{c}, x_{d}\right) \leq(1,1,1,0)\right\} .
$$

Proof of Theorem 5. We define the following concept:

P-justified objection: Let $V$ be a game, $x$ a payoff profile, $(S, y)$ an objection to $x$. Then, $(S, y)$ is $P$-justified if there is no precise and strict counterobjection to $(S, y)$ at $x$.

Let $V$ be a game. For convenience, we identify $\mathcal{P} \cup\{\varnothing\}$ with $\mathcal{P}$ for every coalition structure $\mathcal{P}$, and define $V(\varnothing) \equiv \mathbb{R}^{N}$ and $\{\{i\} \mid i \in \emptyset\} \equiv \emptyset$. Let $x^{0} \equiv r \in \mathbb{R}^{N}$, where $r_{i}=\max V_{\{i\}}$ for all $i \in N$. If there is no P-justified objection to $x^{0}$, then $x^{0} \in P B(V ;\{\{i\} \mid i \in N\})$. Otherwise, go to Stage 1 .

Stage 1. Choose a P-justified objection $\left(S(0), y^{0}\right)$ to $x^{0}$ so that there is no $y^{\prime} \in V_{S(0)}$ such that $y^{\prime} \gg$ $y^{0}$. Consider the coalition structure $\{S(0)\} \cup\{\{i\} \mid i \in N \backslash S(0)\}$, and define $x^{1} \equiv\left(y^{0}, r_{N \backslash S(0)}\right)$. Then, $x^{0} \leq x^{1}, x^{0} \neq x^{1}$ and $x^{1} \in V(S(0)) \cap \bigcap_{i \in N \backslash S(0)} V(\{i\})$. If there is no P-justified objection to $x^{1}$, then $x^{1} \in P B(V ;\{S(0)\} \cup\{\{i\} \mid i \in N \backslash S(0)\})$. Otherwise, go to Stage 2 .

Stage 2. Choose a P-justified objection $\left(S(1), y^{1}\right)$ to $x^{1}$ in the same way as above. Since $x^{0} \leq x^{1}$, $\left(S(1), y^{1}\right)$ is also an objection to $x^{0}$ and strictly improves upon $\left(S(0), y^{0}\right)$ at $x^{0}$. Since the objection $\left(S(0), y^{0}\right)$ to $x^{0}$ is P-justified, we have $S(1) \cap S(0)=\emptyset$ or $S(0) \backslash S(1)=\emptyset$. Consider the coalition structure $\{S(1), S(0) \backslash S(1)\} \cup\{\{i\} \mid i \in N \backslash(S(1) \cup S(0))\}$. The possible features of $\{S(0) \backslash S(1)\}$ are $\{S(0)\}$ and $\{\varnothing\}$. Define $x^{2} \equiv\left(y^{1}, y_{S(0) \backslash S(1)}^{0}, r_{N \backslash(S(1) \cup S(0))}\right)$, where

$$
\left(y_{S(0) \backslash S(1)}^{0}, r_{N \backslash(S(1) \cup S(0))}\right) \equiv\left\{\begin{array}{cc}
\left(y^{0}, r_{N \backslash(S(1) \cup S(0))}\right) & \text { if } S(0) \backslash S(1)=S(0) \\
r_{N \backslash S(1)} & \text { if } S(0) \backslash S(1)=\varnothing
\end{array}\right.
$$

Then, $x^{2} \in V(S(1)) \cap V(S(0) \backslash S(1)) \cap \bigcap_{i \in N \backslash(S(1) \cup S(0))} V(\{i\})$. If there is no P-justified objection, $x^{2} \in P B(V ;\{S(1), S(0) \backslash S(1)\} \cup\{\{i\} \mid i \in N \backslash(S(1) \cup S(0))\})$. Otherwise, go to Stage 3.

$\underline{\text { Stage } 3}$. Choose a P-justified objection $\left(S(2), y^{2}\right)$ to $x^{2}$ in the same way as above. Since $x^{0} \leq x^{2}$, $\left(S(2), y^{2}\right)$ is also an objection to $x^{0}$ and strictly improves upon $\left(S(0), y^{0}\right)$ at $x^{0}$. Since the objection $\left(S(0), y^{0}\right)$ to $x^{0}$ is P-justified, we have $S(2) \cap S(0)=\varnothing$ or $S(0) \backslash S(2)=\varnothing$. Similarly, 
$\left(S(2), y^{2}\right)$ is an objection to $x^{1} \leq x^{2}$ and the objection $\left(S(1), y^{1}\right)$ to $x^{1}$ is P-justified, so that $S(2) \cap S(1)=\varnothing$ or $S(1) \backslash S(2)=\varnothing$. Consider the coalition structure $\{S(2), S(1) \backslash S(2), S(0) \backslash$ $(S(2) \cup S(1))\} \cup\{\{i\} \mid i \in N \backslash(S(2) \cup S(1) \cup S(0))\}$. The possible features of $\{S(1) \backslash S(2), S(0) \backslash$ $(S(2) \cup S(1))\}$ are $\{S(1), S(0)\},\{S(1)\},\{S(0)\}$ and $\{\varnothing\}$.

Define $x^{3} \equiv\left(y^{2}, y_{S(1) \backslash S(2)}^{1}, y_{S(0) \backslash(S(2) \cup S(1))}^{0}, r_{N \backslash(S(2) \cup S(1) \cup S(0))}\right)$, where $\left(y_{S(1) \backslash S(2)}^{1}, y_{S(0) \backslash(S(2) \cup S(1))}^{0}, r_{N \backslash(S(2) \cup S(1) \cup S(0))}\right)$

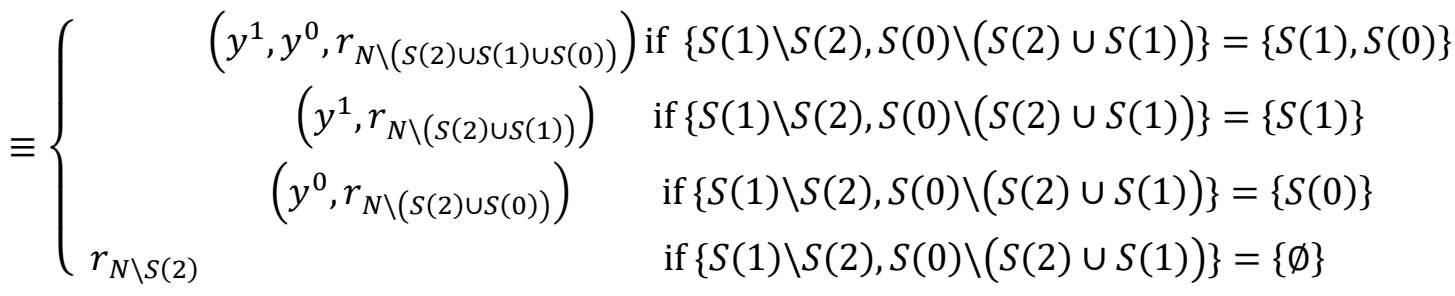

Then, $x^{3} \in V(S(2)) \cap V(S(1) \backslash S(2)) \cap V(S(0) \backslash(S(2) \cup S(1))) \cap \bigcap_{i \in N \backslash(S(2) \cup S(1) \cup S(0))} V(\{i\})$.

If there is no P-justified objection, then

$$
x^{3} \in P B(V ;\{S(2), S(1) \backslash S(2), S(0) \backslash(S(2) \cup S(1))\} \cup\{\{i\} \mid i \in N \backslash(S(2) \cup S(1) \cup S(0))\}) .
$$

Otherwise, go to Stage 4.

Suppose that this process does not terminate at Stage $k-1$. Then we go to Stage $k$.

Stage $k$. For every $t=1, \cdots, k-1,\left(S(t), y^{t}\right)$ is a P-justified objection to

$$
x^{t} \equiv\left(y^{t-1}, y_{S(t) \backslash S(t-1)}^{t-2}, \cdots, y_{S(0) \backslash(S(t-1) \cup \cdots \cup S(1))}^{0}, r_{N \backslash(S(t-1) \cup \cdots \cup S(1) \cup S(0))}\right),
$$

which is chosen in the same way as above.

Note that $S\left(t^{\prime}\right) \cap S(t)=\varnothing$ or $S\left(t^{\prime}\right) \backslash S(t)=\emptyset$ for each $t^{\prime}, t=1, \cdots, k-1$ with $t^{\prime}<t$. For each $t=0,1, \cdots, k-1$, let $U(t) \equiv S(k-1) \cup \cdots \cup S(t)$. Consider the coalition structure $\{S(k-$ 1), $S(k-2) \backslash U(k-1), \cdots, S(0) \backslash U(1)\} \cup\{\{i\} \mid i \in N \backslash U(0)\}$, and define

$$
x^{k} \equiv\left(y^{k-1}, y_{S(k-2) \backslash U(k-1)}^{k-2}, \cdots, y_{S(0) \backslash U(1)}^{0}, r_{N \backslash U(0)}\right) \text {, }
$$

Then

$$
x^{k} \in V(S(k-1)) \cap V(S(k-1) \backslash U(k-1)) \cap \cdots V(S(0) \backslash U(1)) \cap \bigcap_{i \in N \backslash U(0)} V(\{i\}) .
$$

If there is no P-justified objection to $x^{k}$, then

$$
x^{k} \in P B(V ;\{S(k-1), S(k-1) \backslash U(k-1), \cdots, S(0) \backslash U(1)\} \cup\{\{i\} \mid i \in N \backslash U(0)\})
$$

Otherwise, go to Stage $k+1$.

By construction, each coalition can make a P-justified objection at most once. Since the number of coalitions is finite, this process terminates in finite stages. Then, we can find a point of $P B(V ; \mathcal{P})$ for at least one coalition structure $\mathcal{P}$. 


\section{References}

Aumannm R.J. (1989): Lectures on Game Theory, Boulder: Westview Press.

Aumann, R.J. and Drèze, J.H. (1974): "Cooperative games with coalition structures," International Journal of Game Theory, 3, 217-237.

Aumann R.J. and M. Maschler (1964): "The bargaining set for cooperative games," M. Dresher, L.S. Shapley, and A. Tucker., eds., Advances in Game theory, 443-476, New Jersey: Princeton University Press.

Bondareva O.N. (1963): "Some applications of linear programming methods to the theory of cooperative games" (in Russian), Problemy Kibernet, 10, 119-139.

Chang C. and Y.J. Lee (1993): "A non-weakly balanced game with a non-empty bargaining set," Journal of Mathematical Economics 22, 195-198.

Davis M. and M. Maschler (1963): "Existence of stable payoff configurations for cooperative games," Bulletin of the American Mathematical Society, 69, 106-108.

- (1965): "The kernel of a cooperative game," Naval Research Logistics Quarterly, 12, 223-259.

Demuynck T. and T. Potoms (2020): "Weakening transferable utility: The case of non-intersecting Pareto curves," Journal of Economic Theory 188, doi.org/10.1016/j.jet.2020.105035

Iñarra E, R., R. Serrano and K.-I. Shimomura (2020): “The nucleolus, the kernel, and the bargaining set: An update," Revue économique 71, 225-266.

Kakutani S. (1941): "A generalization of Brouwer's fixed point theorem," Duke Mathematical Journal, $8,457-459$

Mas-Colell A. (1987): "Cooperative equilibrium," M. Eatwell, M. Milgate, P. Newmann., eds, The New Palgrave: A Dictionary of Economics, Vol 1, 659-662, London: Macmillan.

- (1989): "An equivalence theorem for a bargaining set," Journal of Mathematical Economics, 18, 129139.

Peleg B. (1963): "Existence theorem for the bargaining set $\mathrm{M}_{1}{ }^{(\mathrm{i})}$," Bulletin of the American Mathematical Society, 69, 109-110.

Peleg B. (1985): "An axiomatization of the core of cooperative games without side payments," Journal of Mathematical Economics, 14, 203-214.

Scarf H. (1967): "The core of an n-person game," Econometrica, 35, 50-69

Shapley L.S. (1967): "On balanced sets and cores," Naval Research Logistics Quarterly, 14, 453-460.

Shimomura K.-I. (1997): "Quasi-cores in bargaining sets," International Journal of Game Theory, 26, 283-302.

Thomson W. (2021): Consistent Allocation Rules, Cambridge: Cambridge University Press, forthcoming.

Vohra R. (1991): "An existence theorem for a bargaining set," Journal of Mathematical Economics, 20, $19-34$.

- (1997): "Coalitional non-cooperative approaches to cooperation," S. Hart, and A. Mas-Colell, Eds., Cooperation: Game Theoretic Approaches, 127-141, Berlin: Springer-Verlag.

Zhou L. (1994): "A new bargaining set of an n-person game and endogenous coalition formation," Games and Economic Behavior, 6, 512-526. 